\title{
Sparse monolithic compliant mechanisms using continuum structural topology optimization
}

\author{
S. Rahmatalla and C. C. Swan*,† \\ Civil and Environmental Engineering, Center for Computer-Aided Design, The University of Iowa, \\ Iowa City, Iowa 52242, U.S.A.
}

\begin{abstract}
SUMMARY
A formulation for design of continuous, hinge-free compliant mechanisms is developed and examined within a continuum structural topology optimization framework. The formulation makes use of two distinctly different sets of springs, the first of which are artificial springs of relatively large stiffness attached to the input and output ports of the mechanism model, and the second of which are springs attached only to the output port with smaller stiffnesses that represent the resistance of the workpiece as it is manipulated by the mechanism. The proposed formulation involves solving two nested optimization problems. In the inner problem the arrangement of a constrained amount of structural material is optimized to maximize the mechanism's mutual potential energy in response to a force loading at the input port while working against the stiff artificial springs on the input and output ports. As the relative stiffness of the artificial springs increases, the material continuity of the mechanism also increases to the point where de facto 'hinge' regions are eliminated. In the outer problem, the artificial springs are removed and one solves for an appropriate amount of structural material that yields the desired finite deformation compliance characteristics of the mechanism when working against the real workpiece resistance. Different aspects of the proposed formulation are demonstrated on a number of examples and discussed. Copyright (C) 2004 John Wiley \& Sons, Ltd.
\end{abstract}

KEY WORDS: MEMS; design optimization

\section{INTRODUCTION}

\subsection{Compliant vs rigid-body mechanisms}

In rigid-body mechanisms, the components of a system work together as essentially inflexible members to convert forces/torques applied at an input port into desired kinematic motion at a designated output port of the system. While rigid-body mechanisms (such as pulleys, hinges, gears, shafts, cranks, etc.) can be optimal in innumerable macroscopic mechanical systems, they are less suited for micro-scale mechanical systems due to the fundamental difficulty

\footnotetext{
${ }^{*}$ Correspondence to: C. C. Swan, Civil and Environmental Engineering, Center for Computer-Aided Design, The University of Iowa, Iowa City, Iowa 52242, U.S.A.

†E-mail: colby-swan@uiowa.edu 
of fabricating reliable hinged- or pinned-joints on such small scales. Accordingly, compliant mechanisms that transmit applied forces/torques from input ports to output ports by elastic deformation of the comprising material are receiving considerable attention for microscale mechanical systems. In compliant mechanisms, the system is generally continuous, monolithic and, to some extent, flexible.

Although methods for fabricating micro- and nano-scale compliant mechanisms (and MEMS) have undergone considerable development over the past decade, corresponding methodologies for design of compliant mechanisms remain an open area. One of the first approaches used for designing compliant mechanisms was based on converting analogous rigid-body mechanisms into compliant mechanisms [1]. While many workable compliant mechanisms have been designed using this approach [2], it can require a good deal of human intuition and involvement to achieve suitable mechanisms with specified performance characteristics.

A second approach for designing compliant mechanisms that accounts for both the stiffness and flexibility requirements of the mechanism involves usage of structural topology optimization. Ananthasuresh et al. [3] were among the first to apply continuum topology optimization to design of compliant mechanisms, using a multi-objective formulation based on weighted sums of the mechanism flexibility and stiffness. Frecker et al. [4] explored continuum topology design of compliant mechanisms using a product of stiffness and flexibility objectives but noted some difficulty in controlling the tradeoffs between flexibility and stiffness. Larsen et al. [5] employed an objective function that used both the mechanical advantage (MA) and geometrical advantage (GA) of the mechanism to achieve compliant mechanism designs with specified force/displacement characteristics.

While the works cited were intended to design single-material mechanisms with modelling based on linear elasticity theory, continuum topology optimization methods for compliant mechanisms have more recently been extended to include geometrical non-linearity [6-9], stress constraints and strength [10,11], multiple materials [12] and multi-physics [13-15]. Despite these significant advances, a number of more fundamental issues still remain to be resolved in order to achieve designs that will function as intended, and so some of these issues are addressed in this manuscript.

\subsection{The challenge of De Facto hinges in design of compliant mechanisms}

One of the main difficulties in applying continuum topology optimization formulations to design of compliant mechanisms is their strong tendency to introduce de facto hinges into the design models, making them function essentially as rigid-body mechanisms [12]. Such de facto hinge zones are typically artifacts of the finite element method used in the analysis and the problem formulation used to obtain the design. A specific illustration of the problem is provided in two fairly typical continuum topology design solutions for a force-inverter compliant mechanism (Figure 1). Both designs shown feature numerous de facto hinge regions which are points in the finite element model about which, when the mechanism is loaded, the surrounding materials (structural members) undergo essentially rigid-body rotations. The de facto hinge regions generally lie along the force path from the mechanism input port to the output port and are zones of high material compliance. Since it is presently very difficult to fabricate reliable hinges for micro-scale mechanical systems, designs that feature de facto hinges are of questionable utility. Accordingly, a variety of strategies have been investigated for dealing with problematic de facto hinge regions in continuum topology design of compliant mechanisms. 


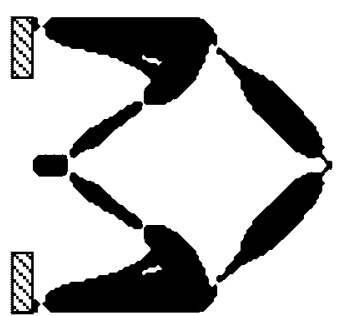

(a)

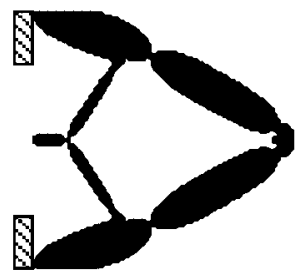

(c)

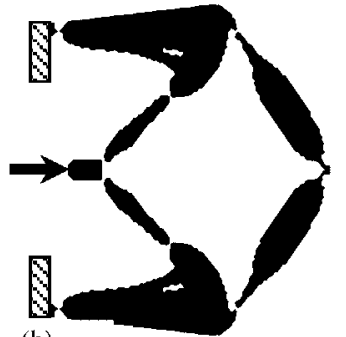

(b)

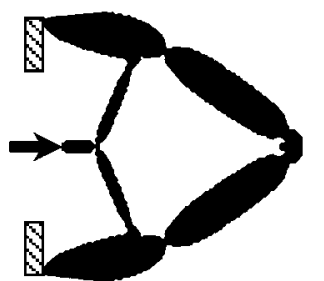

(d)

Figure 1. (a) Undeformed configuration of material layout for a force-inverter compliant mechanism obtained by minimizing a quotient objective function; (b) deformed configuration under loading; (c) undeformed configuration of force-inverter mechanism obtained by minimizing weighted sum objective function; and (d) deformed configuration under loading.

One notable approach to solving the de facto hinge problem is to use second-stage design operations that attempt to redesign the de facto hinge regions as continuous material bridges that function as lumped compliance regions [8]. In such approaches, the lumped compliance regions are modelled as compliant, necked links of material that connect the essentially rigid members of the mechanism. An attempt is then made to design the compliant links to have suitable shape characteristics (length and thickness) such that the maximum strain is below the yield strain of the material and such that the mechanism will still function as desired.

A number of different techniques for eliminating de facto hinges in compliant mechanism models have been investigated by Poulsen who in Reference [16] formulated the continuum topology design problem within a finite element framework and used wavelet basis functions to interpolate material layout design variables. Although the wavelet basis functions were chosen in a way that attempted to preclude formation of de facto hinge regions, the approach was not entirely effective, making necessary second-stage operations to re-design the hinge zones. In a subsequent work Poulsen [17] had more success at elimination of hinges in compliant mechanisms, and mesh-dependent designs in general, by imposing monotonicity based minimum length scale constraints on the designs.

More physically based approaches were investigated by Yin and Ananthasuresh [18] who studied two alternative strategies for obtaining material layout designs free of de facto hinges within a traditional FEM framework. In the first, an upper bound constraint was imposed on the distortional strain energy in the de facto hinge regions, and in the second an upper bound constraint was imposed on local relative material rotations. The first of the two attempted approaches was unsuccessful at precluding lumped compliance regions, while the second approach showed some success in yielding distributed compliance mechanisms. 
A final approach to topological design of compliant mechanisms that circumvents difficulties associated with formation of de facto hinge regions in continuum topology methods involves ground structure methods. These solve for the optimal arrangements of discrete frame members that can carry extensional, shear, and bending loads [9, 19-21]. The joints connecting individual frame members transmit moments and thus do not behave as de facto hinges. Nevertheless, a significant challenge with these approaches is that they do not necessarily model all of the interactions between frame members that cross within the plane of the structure.

\subsection{Scope of article}

In the body of this paper, a novel formulation for design of monolithic, hinge-free compliant mechanisms is investigated within a linear and non-linear elastic continuum topology optimization framework. The formulation makes use of two distinctly different sets of springs, the first of which are artificial springs of relatively large stiffness attached to the input and output ports of the mechanism model, and the second of which are springs attached only to the output port with smaller stiffnesses that represent the resistance of the workpiece as it is manipulated by the mechanism. The proposed formulation involves solving two nested optimization problems. In the inner problem the arrangement of a constrained amount of structural material is optimized to maximize the mechanism's linear elastic mutual potential energy [22] in response to a force loading at the input port while working only against the stiff artificial springs on the input and output ports. As the relative stiffness of the artificial springs increases, the material continuity of the mechanism also increases to the point where de facto 'hinge' regions are eliminated. In the outer problem, the artificial springs are removed and one solves for an appropriate amount of structural material that yields the desired finite deformation compliance characteristics of the mechanism when working against the real workpiece resistance. A number of examples are presented with varying artificial spring stiffnesses, varying structural materials, and varying material usage constraints to investigate the validity and utility of the proposed formulation, followed by discussion and concluding remarks.

Preceding efforts $[3,14,20,23]$ have introduced springs on the output ports of compliant mechanism models to capture the resistances associated with workpieces, while some have introduced constraints on the input port displacements. These springs and displacement constraints facilitate, to some degree, an ability to control the mechanical advantage (MA) and the geometrical advantage (GA) of the mechanism being designed. While springs and displacement constraints have indeed been utilized in previous design studies, a systematic study of their impact on both formation of de facto hinge regions and control of stiffness/flexibility tradeoffs has not been systematically explored. The proposition of this work is that judicious usage of springs in the design of compliant mechanisms can facilitate realization of monolithic compliant mechanism designs and by subsequently manipulating the sparsity of the mechanism designs the compliance characteristics can be tuned to desired levels.

\section{ELEMENTS OF PROBLEM FORMULATION}

\subsection{Design variables, mixing rules, analysis model}

Within a spatial domain $\Omega_{b}$ the layout of structural material with which a compliant mechanism is to be fabricated is here called the 'design' of the mechanism. To achieve a detailed and yet 
finite-dimensional description of material layout in the mechanism within $\Omega_{b}$, a 'design vector' b consisting of $N$ design variables is used

$$
\mathbf{b}=\left\{\phi_{1}, \phi_{2}, \ldots, \phi_{N}\right\}
$$

where each of the design variables assumes a value on the interval $[0,1]$ and denotes a volumetric density of structural material. In the particular framework used here, each design variable in Equation (1) corresponds to volumetric density of structural material at a node in the mathematical model used to compute the structural performance of the mechanism. Accordingly, at a given spatial point $\mathbf{X} \in \Omega_{b}$ the volumetric density of structural material can be obtained simply by interpolation of the nodal densities as follows:

$$
\phi(\mathbf{X})=\sum_{A=1}^{N} N_{A}(\mathbf{X}) \phi_{A}
$$

where the $N_{A}$ represent a set of linearly independent nodal basis functions that feature at least $C^{0}$ continuity. Because this formulation assures $C^{0}$ continuity of the design variable field no precautions need be taken to preclude 'checkerboarding' designs, although the method will generally need to be used with perimeter constraints [24] to ensure convergence of designs with mesh refinement of the analysis model [25].

Typically layout optimization of material in compliant mechanisms is performed with a finite (or constrained) amount of material specified as a fraction $\Im_{M}$ of the mechanism's envelope volume. For a given design $\mathbf{b}$, the fractional volume of structural material as compared to the mechanism's envelope volume is computed as follows:

$$
\mathfrak{\Im}_{\mathrm{M}}=\frac{\int_{\Omega_{b}} \phi(\mathbf{X}) \mathrm{d} \Omega}{\int_{\Omega_{b}} \mathrm{~d} \Omega}
$$

where the numerator provides the material volume of the mechanism, and the denominator the envelope volume of the mechanism model.

Since at each point $\mathbf{X} \in \Omega_{b}$ there is generally a mixture of a solid structural material and a void-like material with respective volume fractions $\phi(\mathbf{X})$ and $1-\phi(\mathbf{X})$ a methodology is generally needed to determine the effective stiffness properties of the solid-void mixture or composite. A number of different possibilities exist, and a fairly detailed review was presented in Reference [26]. Here, a simple iso-deformation powerlaw mixing rule is employed in which it is assumed that both the solid and void-like material at a point $\mathbf{X}$ undergo identical deformations. Accordingly, at each point $\mathbf{X} \in \Omega_{b}$, both the solid and void-like materials share the same deformation gradient:

$$
\mathbf{F}(\mathbf{X})=\mathbf{F}_{\text {solid }}(\mathbf{X})=\mathbf{F}_{\text {void }}(\mathbf{X}) \equiv \frac{\partial \mathbf{x}}{\partial \mathbf{X}}
$$

The particular isotropic hyperelastic strain energy function $U$ used here is that of Ciarlet [27] wherein the volumetric $\left(U_{\mathrm{V}}\right)$ and deviatoric $\left(U_{\mathrm{D}}\right)$ strain energy functions are assumed 
to be decoupled and of the forms:

$$
\begin{aligned}
U(\boldsymbol{F}) & =U_{\mathrm{V}}(J)+U_{\mathrm{D}}(\boldsymbol{\theta}) \\
U_{\mathrm{V}}(J) & =\frac{1}{2} K\left[\frac{1}{2}\left(J^{2}-1\right)-\ln (J)\right] \\
U_{\mathrm{D}} & =\frac{1}{2} \mu[\operatorname{tr}(\overline{\boldsymbol{\theta}})-3]
\end{aligned}
$$

In the preceding expression, $J$ is the determinant of $\boldsymbol{F} ; K$ is a constant bulk modulus; $\mu$ is a constant shear modulus; $\boldsymbol{\theta}=\boldsymbol{F} \boldsymbol{F}^{\mathrm{T}}$ is the left Cauchy-Green deformation tensor; and $\overline{\boldsymbol{\theta}}=J^{-(2 / 3)} \boldsymbol{\theta}$ is its scaled counterpart having a determinant of unity. For this model, therefore, the Kirchhoff stress $\tau$ in a material is thus related to deformation quantities as follows:

$$
\tau=\frac{K}{2}\left(J^{2}-1\right) \mathbf{1}+\mu \mathbf{I}_{\mathrm{dev}}: \overline{\boldsymbol{\theta}}
$$

where $\mathbf{1}$ is the rank-2 identity tensor, and $\mathbf{I}_{\mathrm{dev}}=\mathbf{I}-\frac{1}{3}(\mathbf{1} \otimes \mathbf{1})$ is the rank-4 deviatoric tensor with I the rank-4 identity operator.

Although the solid and void-like materials share the same state of deformation in accordance with Equation (4), the stress states in each are generally consistent with their own constitutive behaviors and material properties. Assuming that both the solid and void-like materials can be represented by the isotropic hyperelastic constitutive model above it follows that a point $\mathbf{X}$ the stresses in the respective materials would be

$$
\begin{aligned}
& \tau_{\text {solid }}=\frac{K_{\text {solid }}}{2}\left(J^{2}-1\right) \mathbf{1}+\mu_{\text {solid }} \mathbf{I}_{\text {dev }}: \overline{\boldsymbol{\theta}} \\
& \tau_{\text {void }}=\frac{K_{\text {void }}}{2}\left(J^{2}-1\right) \mathbf{1}+\mu_{\text {void }} \mathbf{I}_{\text {dev }}: \overline{\boldsymbol{\theta}}
\end{aligned}
$$

In accordance with the powerlaw mixing rule the average stress in the solid-void mixture at point $\mathbf{X}$ is simply the weighted sum as follows:

$$
\tau(\mathbf{X})=\phi^{P}(\mathbf{X}) \tau_{\mathrm{solid}}+\left[1-\phi^{P}(\mathbf{X})\right] \tau_{\mathrm{void}}
$$

To achieve the effect of a void-like material in this work, the bulk and shear moduli of the void material are taken to be $10^{-6}$ times those in the solid structural material. In the mixing rule of Equation (8), the powerlaw exponent $P$ is generally chosen larger than unity, but less than or equal to four. A value of unity yields the classical Voigt rule of mixtures, whereas a value of $P=4$ leads to a penalized mixture in which stiffnesses approaching that of the solid material are achieved only for values of $\phi$ very close to unity.

The strong form of the non-linear elliptic boundary value problem to be solved for the structural displacement field in the compliant mechanism model is as follows:

Find $\mathbf{u}:\left(\Omega_{b} \times[0, T]\right) \mapsto \mathfrak{R}^{3}$ such that:

$$
\tau_{i j, j}+\rho_{0} \gamma_{j}=0 \quad \text { on } \boldsymbol{\Omega}_{b} \forall t \in[0, T]
$$


subject to the boundary conditions:

$$
\begin{aligned}
& u_{j}(t)=g_{j}(t) \text { on } \Gamma_{g j} \quad \text { for } j=1,2,3, \quad \forall t \in[0, T] \\
& n_{i} \tau_{i j}=h_{j}(t) \text { on } \Gamma_{h j} \quad \text { for } j=1,2,3, \quad \forall t \in[0, T]
\end{aligned}
$$

Above, $\tau$ denotes the Kirchhoff stress tensor field which is related to the Cauchy stress tensor $\boldsymbol{\sigma}$ via the relation $\tau=J \boldsymbol{\sigma}$, where $J=\operatorname{det}(\mathbf{F})$ and $\mathbf{F}$ is the deformation gradient operator. As is customary, it is assumed that the Lagrangian surface $\boldsymbol{\Gamma}=\overline{\boldsymbol{\Gamma}_{g j} \cup \boldsymbol{\Gamma}_{h j}}$ bounding the Lagrangian structural domain $\Omega_{b}$ admits the decomposition $\Gamma_{g_{j}} \cap \Gamma_{h_{j}}=\{\emptyset\}$ for $j=1,2$,3. For a given mesh discretization of $\Omega_{b}$ whose complete set of nodes is denoted $\eta$, the subsequent design formulation is facilitated by introducing a subset of nodes $\eta_{h}$ at which non-vanishing external forces are applied, and a subset of nodes $\eta_{g}$ at which non-vanishing prescribed displacements are applied. The nodes in the model at which the unknown displacements remain to be determined form the set denoted $\eta-\eta_{g}$.

Using standard techniques, the virtual work equivalent of the original problem statement in Equations (9) can be obtained in the following form:

$$
\int_{\Omega_{b}} \tau_{i j} \delta \varepsilon_{i j} \mathrm{~d} \Omega_{S}=\int_{\Omega_{b}} \rho_{0} \gamma_{j} \delta u_{j} \mathrm{~d} \Omega_{S}+\int_{\Gamma_{h}} h_{j} \delta u_{j} \mathrm{~d} \Gamma_{h}
$$

In the expression above, the quantity on the left represents the internal virtual work $\delta W^{\text {int }}$, and that on the right, the external virtual work $\delta W^{\text {ext }}$.

Usage of a Galerkin formulation, in which the real and variational kinematic fields are expanded in terms of the same nodal basis functions, and discretization of the time domain into a finite number of discrete time points, leads to the following force balance equations at each unrestrained node $\mathrm{A}$ in the mesh as here at the $(n+1)$ th time step:

$$
\mathbf{r}_{n+1}^{A}=\left(\mathbf{f}^{\mathrm{int}}\right)_{n+1}^{A}-\left(\mathbf{f}^{\mathrm{ext}}\right)_{n+1}^{A}=\mathbf{0} \quad \forall A \in \eta-\eta_{g}
$$

where

$$
\begin{aligned}
\left(\mathbf{f}^{\text {int }}\right)_{n+1}^{A}=\int_{\Omega_{b}}\left(\mathbf{B}^{A}\right)_{n+1}^{T}: \tau_{n+1} \mathrm{~d} \Omega_{b} \\
\left(\mathbf{f}^{\mathrm{ext}}\right)_{n+1}^{A}=\int_{\Omega_{b}} \rho_{0} N^{A} \gamma_{n+1} \mathrm{~d} \Omega_{b}+\int_{\Gamma_{h}} N^{A} \mathbf{h}_{n+1} \mathrm{~d} \Gamma_{h}
\end{aligned}
$$

In Equation (12), $\mathbf{B}_{n+1}^{A}$ represents the spatial infinitesimal nodal strain displacement matrix $\left(\mathbf{B}_{n+1}^{A}=\nabla_{x_{n+1}}^{s} N^{A}(\mathbf{x})\right)$, and $N^{A}$ denotes the nodal basis function for the $A$ th node. Under finite deformations, Equation (11) represents a set of non-linear algebraic equations that must be solved in an iterative fashion for the incremental nodal displacements $\left(\Delta \boldsymbol{u}^{A}\right)_{n+1}=$ $\boldsymbol{u}_{n+1}^{A}-\boldsymbol{u}_{n}^{A}$ for each time step of the analysis problem and $\forall A \in \eta-\eta_{g}$. When external forces applied to a structure are independent of its response, the derivative of the $i$ th residual force vector component at the $A$ th node with respect to the $j$ th displacement vector component of 
the $B$ th node is simply:

$$
\mathbf{K}_{i l}^{A B}=\int_{\Omega_{b}} B_{j i}^{A} c_{j k} B_{k l}^{B} \mathrm{~d} \Omega_{S}+\int_{\Omega_{b}} N_{, j}^{A} \tau_{j k} N_{, k}^{B} \delta_{i l} \mathrm{~d} \Omega_{S}
$$

where $c_{j k}$ is the spatial elasticity tensor in condensed form. Assembly of this nodal stiffness operator for all unrestrained nodes $A$ and $B$ gives the structural tangent stiffness matrix. Further implementational details on the powerlaw mixing rule for two hyperelastic solids in a finite deformation framework are available in References [28, 29].

\subsection{Compliant mechanism performance measures}

Within a continuum topology optimization framework, design of compliant mechanisms can be formulated in a number of alternative ways through utilization of assorted objective and constraint functions. Generally, however, the broad objective is to find a compliant mechanism that efficiently converts applied forces/displacements at an input port into desired forces/displacements at an output port, while a constraint function limits the amount of structural material that can be used.

The flexibility and stiffness characteristics of a mechanism can be quantified using relationships between the applied actuation forces, the resulting displacements at the input port of the mechanism, and the resulting displacements and reaction forces at the output port of the mechanism. Larsen et al. [5], for example, used the concepts of the mechanism's GA and MA to quantify the performance characteristics. The GA of a mechanism is defined [Equation $\left(15_{1}\right)$ ] as the component of displacement $\boldsymbol{u}_{\text {out }}$ in a desired direction $\ell_{\text {out }}$ at the output port due to an input port force $\mathbf{f}_{\text {in }}$ divided by the displacement magnitude of the input port $\boldsymbol{u}_{\text {in }}$ also due to the input port force $\mathbf{f}_{\text {in }}$. The MA is defined [Equation $\left(15_{2}\right)$ ] as the ratio of the magnitudes of the reaction force $\boldsymbol{R}$ at the output port and the input port force $\mathbf{f}_{\mathrm{in}}$. The mechanical efficiency (ME) of a mechanism is quantified [Equation (153)] as the product of its GA and MA.

$$
\mathrm{GA}=\frac{\ell_{\text {out }} \cdot \mathbf{u}_{\text {out }}}{\left\|\mathbf{u}_{\text {in }}\right\|} ; \quad \mathrm{MA}=\frac{\mathbf{R} \cdot \ell_{\text {out }}}{\left\|\mathbf{f}_{\text {in }}\right\|} ; \quad \mathrm{ME}=\mathrm{MA} \cdot \mathrm{GA}
$$

The mutual potential energy (MPE) of a mechanism [22] is yet another performance measure that in accordance with Figure 2(a) can be expressed as

$$
\mathrm{MPE}=\mathbf{f}_{\text {out }}^{\mathrm{v}} \cdot \mathbf{u}_{\text {out }}^{(1)}
$$

where $\mathbf{u}_{\text {out }}^{(1)}$ is the displacement at the output port due to the load $\mathbf{f}_{\text {in }}$ applied at the input port, and $\mathbf{f}_{\text {out }}^{\mathrm{v}}$ is a virtual force at the output port specifying the direction of the desired output port displacement. For a given mechanism and input load $\mathbf{f}_{\text {in }}$ the resulting MPE will typically be inversely related to the resistance supplied at the O/P. Hence when one refers to specific values of MPE both the input load $\mathbf{f}_{\text {in }}$ and the O/P resistance should generally be stated. An additional quantity worth noting is termed the complimentary compliance of the mechanism here denoted by $E_{C}$. As used herein the complimentary compliance $E_{C}$ of a mechanism is similar to the MPE with the exception that it explicitly takes account of the input force magnitude:

$$
E_{C}=\frac{\left\|\mathbf{f}_{\text {in }}\right\|}{\left\|\mathbf{f}_{\text {out }}^{\mathrm{v}}\right\|}\left(\mathbf{f}_{\text {out }}^{\mathrm{v}} \cdot \mathbf{u}_{\text {out }}^{(1)}\right)=\frac{\left\|\mathbf{f}_{\text {in }}\right\|}{\left\|\mathbf{f}_{\text {out }}^{\mathrm{v}}\right\|} * \text { MPE }
$$



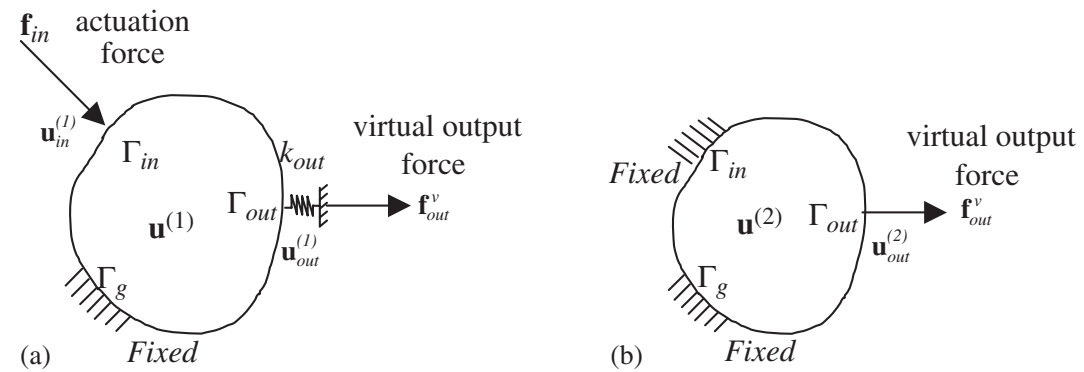

Figure 2. Elements of the compliant mechanism design formulation: (a) problem for analysing kinematic performance of the mechanism (no springs shown attached to $\Gamma_{\text {in }}$ although there generally will be); and (b) problem for computing the input-restrained compliance.

Like the MPE, this quantity, for a given mechanism, will typically be dependent upon the workpiece resistance $k_{\text {out }}$ supplied at the $\mathrm{O} / \mathrm{P}$.

Two last performance measures to be mentioned are the standard mean compliance of the mechanism $\Pi^{(1)}$ when the input port is free and a force $\mathbf{f}_{\text {in }}$ is applied to the input port, and the compliance $\Pi^{(2)}$ of the mechanism when the input port is restrained and a force $\mathbf{f}_{\text {out }}^{\mathrm{v}}$ is applied to the output port. When the mechanism behaves in a linear elastic fashion, the mean compliance $\Pi^{(1)}$ is simply $\Pi^{(1)}=\mathbf{f}_{\text {in }} \cdot \mathbf{u}_{\text {in }}^{(1)}$ where $\mathbf{u}_{\text {in }}^{(1)}$ is the resulting displacement at the input port. The compliance of the mechanism for the loading case (Figure 2(b)) where the input port is restrained and the force $\mathbf{f}_{\text {out }}^{\mathrm{v}}$ is applied to the output port is denoted $\Pi^{(2)}$ and is computed as follows:

$$
\Pi^{(2)}=\mathbf{f}_{\text {out }}^{\mathrm{v}} \cdot \mathbf{u}_{\text {out }}^{(2)}
$$

where $\mathbf{u}_{\mathrm{out}}^{(2)}$ is the resulting displacement at the output port. In subsequent discussion, this quantity is referred to as the 'input-restrained compliance.'

Various combinations of the assorted performance metrics introduced above have been used in design optimization of compliant mechanisms to achieve the desired stiffness and the flexibility characteristics. One notable objective function that has been examined [4] is the ratio of the sign-inverted mutual potential energy and the 'input-restrained compliance:'

$$
\mathfrak{I}=\frac{-\mathrm{MPE}}{\Pi^{(2)}}=\frac{-\mathbf{f}_{\mathrm{out}}^{\mathrm{v}} \cdot \mathbf{u}_{\mathrm{out}}^{(1)}}{\mathbf{f}_{\mathrm{out}}^{\mathrm{v}} \cdot \mathbf{u}_{\mathrm{out}}^{(2)}}
$$

Design optimization of compliant mechanisms (e.g. the force-inverter compliant mechanism in Figure 1(a) and (b)) using this objective function can suffer from the existence of many local minima associated with extremization of the numerator, and extremization of the denominator. Furthermore, this formulation does not appear to preclude the formation de facto hinges. An alternative objective function that permits greater control of the competing stiffness and flexibility objectives is a weighted sum of the mechanism's GA and MA (Figure 1(c) and (d)). Nevertheless, as the design of Figure 1(c) and (d) suggests this formulation can yield problematic designs with de facto hinge regions. 


\section{ENERGY CONSIDERATIONS FOR COMPLIANT MECHANISMS}

Assume that a compliant mechanism is to be designed and that an artificial spring with stiffness $k_{\text {in }}$ is attached to the input port as shown in Figure 3, and an artificial spring with stiffness $k_{\text {out }}$ is attached to the output port. For an actuation force $\mathbf{f}_{\text {in }}$ applied to the input port it is assumed that a minimal energy displacement solution exists satisfying the following equilibrium condition:

$$
\mathbf{f}_{\text {in }}+\mathbf{f}_{\text {in }}^{\text {spring }}+\mathbf{f}_{\text {in }}^{\text {reaction }}=\mathbf{0}
$$

where $\mathbf{f}_{\text {in }}^{\text {spring }}$ is the spring reaction force at the input port, and $\mathbf{f}_{\text {in }}^{\text {reaction }}$ is the reaction force at the input port from the compliant mechanism system. The total work done by the actuation force at the input port $\left(W_{\text {in }}\right)$ can be written as follows:

$$
W_{\text {in }}=W_{\text {in }}^{\text {spring }}+W_{\text {in }}^{\text {reaction }}
$$

where $W_{\text {in }}^{\text {spring }}$ is the energy stored in the spring and $W_{\text {in }}^{\text {reaction }}$ that stored in the mechanism. The spring on the input port is associated with a given node (or nodes) of the structural model, and features both a direction and magnitude as follows:

$$
\mathbf{K}_{\text {in }}^{\text {spring }}=k_{\text {in }}\left(\boldsymbol{\delta}_{\text {in }} \otimes \boldsymbol{\delta}_{\text {in }}\right)
$$

where $k_{\text {in }}$ gives the magnitude of the artificial input port spring stiffness, and $\boldsymbol{\delta}_{\text {in }} \in \Re^{\text {ndof } \times \text { numnp }}$ is a unit vector that defines the node(s) and directionality of the input port spring.

If the mechanism design is much more compliant than artificial spring attached to the input port, then most of the input force $\mathbf{f}_{\text {in }}$ will be carried by the spring on the input port,

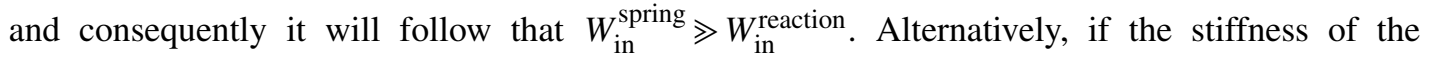

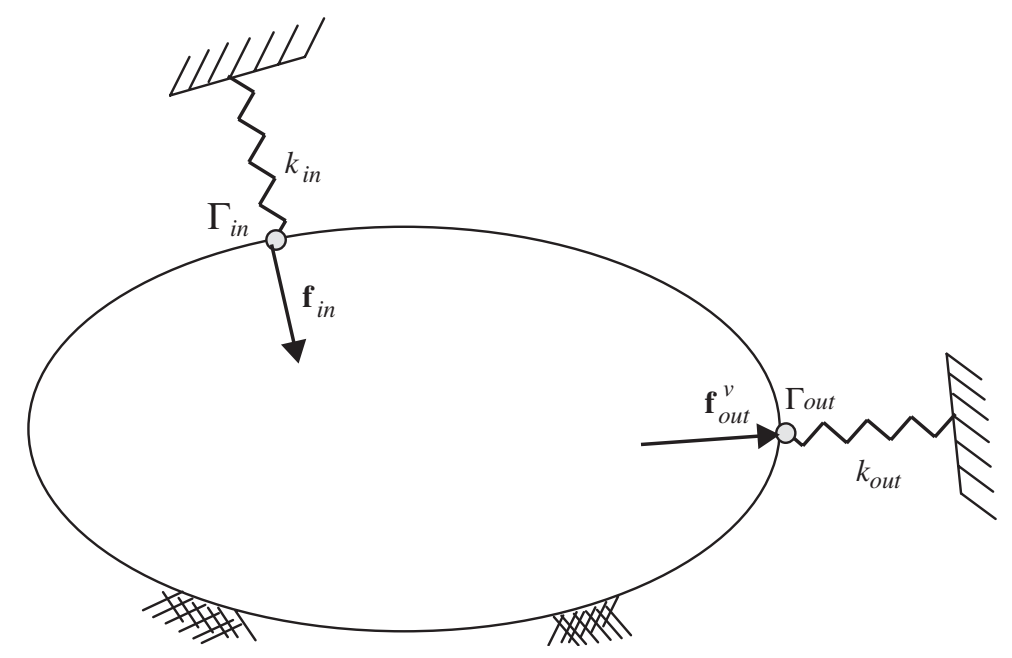

Figure 3. Schematic of compliant mechanism design problem using artificial springs attached to both input port and output port. 
mechanism design approaches that of the artificial input spring $k_{\text {in }}$ a significantly larger fraction of the input force will be transferred to the mechanism, and the energy transferred to the mechanism $W_{\text {in }}^{\text {reaction }}$ will be maximized. This last point is demonstrated quantitatively through brief consideration of a load $f$ carried by two springs of stiffness $k_{s}$ and $\alpha k_{s}$ with $k_{s}>0$ and $\alpha>0$ acting in parallel (Figure 4). The upper spring $\left(k_{s}\right)$ represents the stiffness of an artificial spring attached to the input port, and the lower spring stiffness $\left(\alpha k_{s}\right)$ the input stiffness of the mechanism. For a given aligned force $f$ passing through the parallel spring system, the energy stored in the lower spring (i.e. the mechanism) is denoted by $\alpha / 2 k_{s}(f /(1+\alpha))^{2}$ and this quantity is maximized when $\alpha=1$. The point of this analogy is that the energy transmitted to the mechanism ( $W_{\text {in }}^{\text {reaction }}$ ) is maximized as the stiffness of the mechanism at the I/P approaches that of the artificial spring on the input port $\left(k_{\text {in }}\right)$.

At the output port of the mechanism there is no actuation force and the following equilibrium state must be satisfied:

$$
\mathbf{f}_{\text {out }}^{\text {spring }}+\mathbf{f}_{\text {out }}^{\text {reaction }}=\mathbf{0}
$$

and all of the mechanical work done at the output port will be stored in the attached spring so that:

$$
W_{\text {out }}^{\text {spring }}=W_{\text {out }}^{\text {reaction }}
$$

The artificial spring attached to the output port can be represented by the expression

$$
\mathbf{K}_{\text {out }}^{\text {spring }}=k_{\text {out }}\left(\boldsymbol{\delta}_{\text {out }} \otimes \boldsymbol{\delta}_{\text {out }}\right)
$$

where $k_{\text {out }}$ gives the magnitude of the stiffness, and $\boldsymbol{\delta}_{\text {out }} \in \Re^{\text {ndof } \times \text { numnp }}$ is a unit vector that defines the node(s) and directionality of the spring. The work done at the output port ( $W_{\text {out }}^{\text {spring }}=W_{\text {out }}^{\text {reaction }}$ ) is bounded above by the energy transmitted to the mechanism at the input port, (i.e. $W_{\text {in }}^{\text {reaction }} \geqslant W_{\text {out }}^{\text {reaction }}$ ). In fact, the difference between $W_{\text {in }}^{\text {reaction }}$ and $W_{\text {out }}^{\text {reaction }}$ is the energy stored in the mechanism $W_{\text {stored }}^{\text {mechansm }}$. Consequently, if the design goal is to maximize $W_{\text {out }}^{\text {reaction }}$ this can be done by first maximizing $W_{\text {in }}^{\text {reaction }}$ and then minimizing the strain energy stored in the mechanism $W_{\text {stored }}^{\text {mechanism }}$.

To summarize these observations regarding effects of input and output port spring stiffnesses:

1. Of the actual energy supplied to the mechanism at the input port ( $\left.W_{\text {in }}^{\text {reaction }}\right)$, only a fraction $\left(W_{\text {stored }}^{\text {mechanism }}\right)$ is stored in the device as strain energy with the remainder ( $W_{\text {out }}^{\text {reaction }}$ ) available to do work against the artificial springs at the O/P. The mechanism design should be fairly stiff to minimize ( $W_{\text {stored }}^{\text {mechanism }}$ ).

2. In design optimization of the mechanism to maximize $W_{\text {out }}^{\text {reaction }}$, the device stiffness near the I/P tends to mimic that of the artificial spring $k_{\text {in }}$. If $k_{\text {in }}$ is small, then the stiffness of the mechanism near the $\mathrm{I} / \mathrm{P}$ will be small, and vice versa.

3. In design optimization of the mechanism to maximize $W_{\text {out }}^{\text {reaction }}$, the device stiffness near the $\mathrm{O} / \mathrm{P}$ tends to mimic that of the artificial spring $k_{\text {out }}$.

(a) With compliant artificial springs attached to the $\mathrm{O} / \mathrm{P}$, the magnitude of $\mathbf{f}_{\text {out }}^{\text {spring }}$ will be relatively small and ( $W_{\text {out }}^{\text {reaction }}$ ) is maximized by increasing the magnitude of $\mathbf{u}_{\text {out }}$. Accordingly, the mechanism will be very compliant in the vicinity of the output port. 


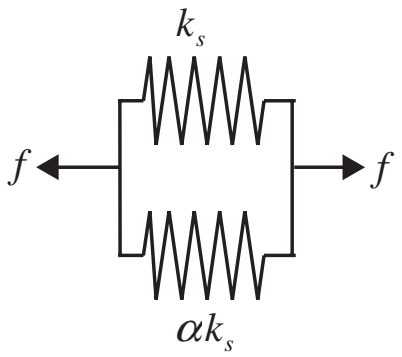

Figure 4. Two springs acting in parallel to carry an applied force $f$. Energy stored in the lower spring is maximized when $\alpha=1$ so that the upper and lower springs have identical stiffnesses.

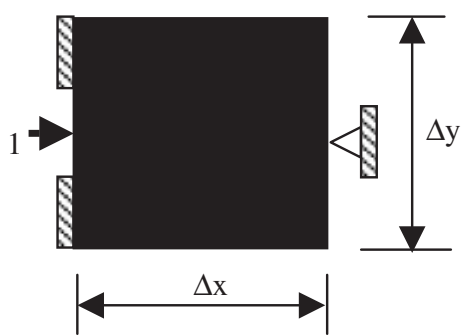

Figure 5. Boundary value problem to determine appropriate bounding stiffnesses $k_{b}$ for artificial springs for the force-inverter mechanism. In the problem, the design domain with $\Delta x=3 \mathrm{~cm}$ and $\Delta y=3 \mathrm{~cm}$ is completely occupied by structural material, the $\mathrm{O} / \mathrm{P}$ is restrained, and a unit load is applied to the I/P. With structural material of aluminium, $k_{b}=1.6 \times 10^{10} \mathrm{~N} / \mathrm{m}$; with polysilicon $k_{b}=4.4 \times 10^{10} \mathrm{~N} / \mathrm{m}$; with nylon $k_{b}=6.9 \times 10^{8} \mathrm{~N} / \mathrm{m}$.

(b) Conversely, with stiffer artificial springs attached to the $\mathrm{O} / \mathrm{P}$, the reaction force $\mathbf{f}_{\text {out }}^{\text {spring }}$ will be larger, and output port work is maximized by creating a large reaction force there together with a modest output displacement. To generate a large reaction force at the $\mathrm{O} / \mathrm{P}$, the stiffness of the mechanism in the vicinity of the $\mathrm{O} / \mathrm{P}$ will tend to match $k_{\text {out }}$.

Based on these observations it is postulated here that mechanism designs containing de facto hinge regions are ineffective at transmitting energy from an input port to an output port when working against stiff artificial springs on both the I/P and O/P (Figure 3). It follows from this postulate that if the mechanism with a set of artificial springs on both the $\mathrm{I} / \mathrm{P}$ and $\mathrm{O} / \mathrm{P}$ as indicated in Figure 3, and for a given actuation force $\mathbf{f}_{\text {in }}$, is designed to maximize $W_{\text {out }}^{\text {reaction }}$, the mechanism stiffness will tend to match those of the artificial springs attached ${ }^{\ddagger}$ and the mechanism design itself will not contain de facto hinges. The objective of this work is to exploit this tendency and in doing so, to obtain functional compliant mechanism designs that are free of de facto hinge regions.

In selecting the artificial spring stiffnesses $k_{\text {in }}$ and $k_{\text {out }}$ to be used in designing a mechanism, appropriate magnitudes should be selected. It is proposed here that upper bounds on $k_{\text {in }}$ and $k_{\text {out }}$ should correspond to the mechanism's I/P and O/P stiffnesses when the design region $\Omega_{b}$ is fully occupied by structural material. Such upper bounds will naturally depend functionally on the size of the mechanism being designed, the stiffness of the material being used to fabricate the mechanism, and the support conditions applied to the mechanism domain $\Omega_{b}$. To account for these factors, upper bound values $k_{b}$, hereafter called bounding spring values on $k_{\text {in }}$ and

\footnotetext{
†If the mechanism design is sparse, with significant constraints on the quantity of material that can be used, then mechanism stiffness will not be able to match that of stiff artificial springs.
} 
$k_{\text {out }}$, are computed by applying a unit load to the mechanism model I/P in the direction of the design loading with the O/P restrained and the structural domain $\Omega_{b}$ fully occupied by the structural material (Figure 5). The stiffness of the model under these conditions provides the bounding spring value $k_{b}$ as follows: $k_{b}=\left\|\mathbf{f}_{\text {in }}\right\| /\left\|\mathbf{u}_{\text {in }}\right\|$ where $\mathbf{u}_{\text {in }}$ is the resulting displacement at the $\mathrm{I} / \mathrm{P}$ when a unit load $\mathbf{f}_{\text {in }}$ is applied there.

\section{A PROPOSED DESIGN FORMULATION}

In the proposed design formulation, usage is made of two distinctly different sets of springs having different purposes. First, the stiff artificial springs discussed above are attached to both the $\mathrm{I} / \mathrm{P}$ and $\mathrm{O} / \mathrm{P}$ of the mechanism with the goal of achieving mechanism designs that, for a fixed amount of structural material, maximize the linear elastic MPE while working against the springs (Figure 6(a)). It is emphasized that such designs, for a specified amount of structural material, will have maximal stiffness in accordance with the energy considerations discussed above. To subsequently model how such mechanism designs function at finite deformations under real workpiece resistance, the stiff artificial springs are removed and a second set of springs, hereafter termed workpiece springs, are used (Figure 6(b)). These workpiece springs are typically attached only to the $\mathrm{O} / \mathrm{P}$ of the mechanism, and are generally much smaller in magnitude than the artificial springs. A realistic goal in design of compliant mechanisms is to have the mechanism be free of de facto hinges, and to have a complimentary compliance $E_{C}$ at finite deformation that exceeds a certain threshold value $E_{C}^{*}$ when working against the workpiece resistance in response to a specified actuation force $\mathbf{f}_{\text {in }}$.

To design a mechanism within the proposed framework that maximizes the linear elastic MPE, a mathematical mechanism model on a spatial region $\Omega_{b} \in \mathfrak{R}^{3}$ is first created and support conditions are prescribed. An input port region $\Gamma_{\text {in }}$ to which an input force $\mathbf{f}_{\text {in }}$ will be applied is identified, as is an output port region $\Gamma_{\text {out }}$ at which $\mathbf{u}_{\text {out }}$ will be monitored. Before the design problem is solved, one also chooses: (1) the structural material; (2) the amount (volume) of structural material that will be used in the design as a fraction $C$ of the design domain spatial volume; and (3) the stiffnesses of the artificial springs that will be attached to both the $\mathrm{I} / \mathrm{P}$ and the $\mathrm{O} / \mathrm{P}$ in accordance with Figure 5. Having made these selections, the material layout in the mechanism that minimizes the sign inverse of the linear elastic MPE under a given actuation force $\mathbf{f}_{\text {in }}$ is obtained as the solution of the following optimization problem subject to a material usage constraint and existence of an equilibrium solution of the structural equilibrium problem:

P1: For fixed material usage constraint value $C$ and artificial springs $\left(k_{\text {in }}, k_{\text {out }}\right)$ :

$$
\min _{\mathbf{b}}[(-\mathrm{MPE})]
$$

such that

$$
\begin{array}{r}
\Im_{M}(\mathbf{b})-C \leqslant 0 \\
\mathbf{r}(\mathbf{u}, \mathbf{b})=\mathbf{0}
\end{array}
$$



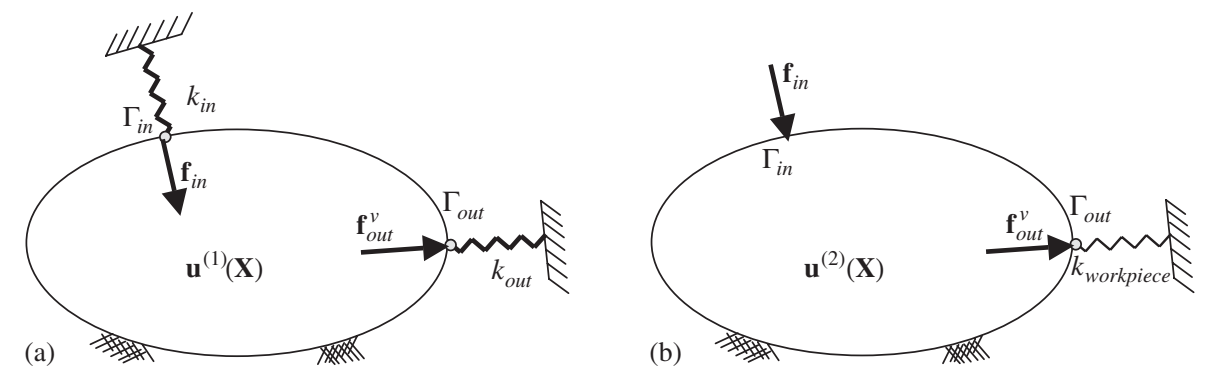

Figure 6. Schematic of analysis cases used to design hinge-free compliant mechanism: (a) Case 1 conditions used to solve problem P1 with linear elastic analysis; and (b) Case 2 conditions solved with geometrically non-linear analysis in problem P2.

where $\mathbf{r} \in \mathfrak{R}^{\text {ndof } \times \text { numnp }}$ is the residual force vector for the linearly elastic structural model and which vanishes when the structure is in equilibrium under the applied actuation force and the spring reaction forces.

If stiff artificial springs $\left(k_{\text {in }}=k_{\text {out }}=k_{b}\right)$ are used in solving design problem P1 posed above, the resulting design solutions will tend to have maximal stiffnesses subject to the material usage constraint value $C$ employed and will be free of de facto hinges. If $\mathrm{P} 1$ is solved a number of times with progressively decreasing values of $C$, the resulting non-linear complimentary compliance values $E_{C}$ of the designs under Case 2 analysis conditions (Figure 6(b)) working against workpiece springs will tend to increase progressively. If the objective is to find the design b that solves P1 with the largest value of the material usage constraint value $C$ for which $E_{C}=E_{C}^{*}$ then a concise mathematical statement of the extended design optimization problem $\mathrm{P} 2$ is as follows:

$\mathrm{P} 2$ : For specified artificial springs $\left(k_{\mathrm{in}}, k_{\mathrm{out}}\right)$ and workpiece springs $k_{\mathrm{workpiece}}$ find:

$$
\begin{gathered}
\inf C \in(0,1) \text { and } \min _{b \in \Re^{N}}(-\mathrm{MPE}) \text { such that: } \\
\Im_{M}-C \leqslant 0 ; \text { material usage constraint } \\
\mathbf{r}\left(\mathbf{u}^{(1)}, \mathbf{b}, C\right)=\mathbf{0} ; \text { equilibrium state in Case } 1 \text { analysis } \\
\mathbf{r}\left(\mathbf{u}^{(2)}, \mathbf{b}, C\right)=\mathbf{0} ; \text { equilibrium state in Case } 2 \text { analysis } \\
E_{C}^{*}-E_{C}\left(\mathbf{u}^{(2)}, \mathbf{b}, C\right) \leqslant 0 ; \text { finite deformation Case } 2 \text { compliance }
\end{gathered}
$$

In P2, the Case 1 analysis (Figure 6(a)) has stiff springs attached to both the I/P and O/P of the structural model, the structure is analysed using linear elastic analysis, and the resulting displacement field in the structural model from which MPE is computed is denoted $\mathbf{u}^{(1)}$. In Case 2 analysis (Figure 6(b)), the stiff springs are removed from the model's I/P and O/P and moderate workpiece springs are attached to the O/P. The finite deformation hyperelastic response of the structure $\mathbf{u}^{(2)}$ to the actuation force $\mathbf{f}_{\text {in }}$ is computed, from which the complimentary compliance $E_{C}\left(\mathbf{u}^{(2)}, \mathbf{b}, C\right)$ is also computed as follows:

$$
E_{C}\left(\mathbf{u}^{(2)}, \mathbf{b}, C\right)=\frac{\left\|\mathbf{f}_{\text {in }}\right\|}{\left\|\mathbf{f}_{\text {out }}^{\mathrm{v}}\right\|}\left(\mathbf{f}_{\text {out }}^{\mathrm{v}} \cdot \mathbf{u}_{\text {out }}^{(2)}\right)
$$


In Equation (28e) of $\mathrm{P} 2, E_{C}^{*}$ is the target value for complimentary compliance when working against the workpiece springs under actuation force $\mathbf{f}_{\text {in }}$ (Figure 6(b)). The approach taken herein to solve P2 is to first solve design problem P1 for numerous values of the material usage constraint $C$ and to then line search for the infimum value of $C$ that satisfies the constraint of Equation (28e). Each of the P1 optimal designs for different $C$ values is analysed at finite deformation under the Case 2 conditions (Figure 6(b)) and complimentary compliances $E_{C}\left(\mathbf{u}^{(2)}, \mathbf{b}, C\right)$ are computed by Equation (29). The design $\mathbf{b}$ associated with the material usage constraint value $C$ that just yields the target complimentary compliance $E_{C}^{*}$ is then taken as the optimum design.

\section{DEMONSTRATIVE EXAMPLES}

\subsection{General features}

Many examples of compliant mechanisms such as, inverting, gripping, and crunching mechanisms have been presented and demonstrated in the design methods literature. The goal of this work is to study the effects of springs and material resource constraints on the continuity and performance characteristics of compliant mechanism designs obtained by solving problem P1 of Equation (26). This is done here in the context of test computations performed on the force-inverter and gripper compliant mechanism design problems. Such compliant mechanisms can be designed and fabricated with a wide variety of materials and here the main materials considered are aluminium $(E=73 \mathrm{GPa} ; v=0.35)$, polysilicon $(E=206 \mathrm{GPa} ; v=0.26)$ and nylon $(E=3 \mathrm{GPa} ; v=0.4)$. In all the examples solved below the initial starting designs utilized a completely solid structural domain $\Omega_{b}$ and the power-law mixing rule (Equation (8)) with $p=4$ was used in the computations. The nodal design variable formulation of Section 2 was employed without any spatial filtering of design variables and a sequential linear programming (SLP) algorithm that used first-order design gradient information to solve all of the example problems.

\subsection{Inverter mechanism designs}

5.2.1. Effect of spring stiffnesses. The function of this device is to have the output port displace in a direction opposite to that of an input force applied at the input port. Figure 7(a) shows the design domain $\Omega_{b}$ of the inverter problem with partial fixed support boundaries at the left hand side. The domain, which is discretized with a $100 \times 100$ bilinear quadrilateral finite element model, is loaded with $f_{\text {in }}=100 \mathrm{~N}$ applied to the input port. The deflection at the output port in the direction of $\mathbf{f}_{\text {out }}^{\mathrm{v}}$ as shown in Figure 7(a) is to be maximized. Before solving this design problem, bounding values for the artificial springs attached to the input and output ports were obtained as described at the end of Section 3 and in Figure 5. Accordingly, the bounding stiffness value obtained for the artificial spring was: $k_{b}=1.6 \times 10^{10} \mathrm{~N} / \mathrm{m}$ for a structural material of aluminium; $k_{b}=4.4 \times 10^{10} \mathrm{~N} / \mathrm{m}$ for polysilicon; and $k_{b}=6.9 \times 10^{8} \mathrm{~N} / \mathrm{m}$ for nylon.

The set of design computation results presented here is intended to highlight the effect of varying the stiffness of the artificial springs attached to input and the output ports of the device (Figure 7(a)) on the resulting design solutions of problem P1. The design material had the elastic properties of aluminium, and a material usage constraint value of $C=0.3$ was 


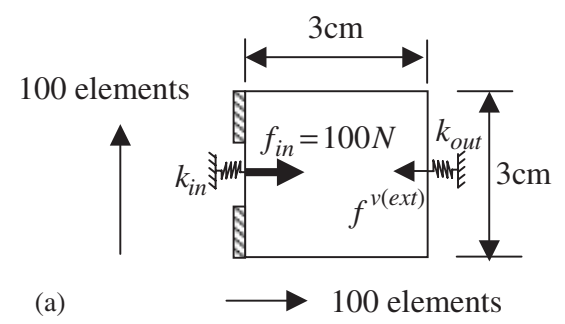

(b)

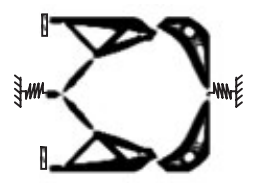

$k_{\text {in }}=k_{b} \times 10^{-4} \quad k_{\text {out }}=k_{b} \times 10^{-4}$

(e)

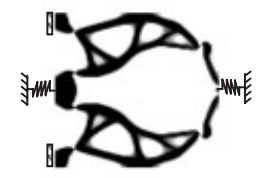

$k_{\text {in }}=k_{b} \times 10^{-2} \quad k_{\text {out }}=k_{b} \times 10^{-4}$

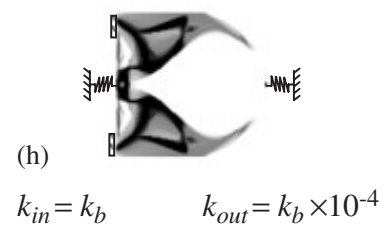

(c)

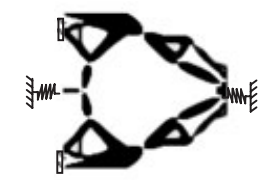

(d)

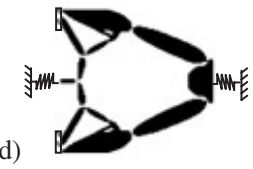

$k_{\text {in }}=k_{b} \times 10^{-4} \quad k_{\text {out }}=k_{b}$

(g)

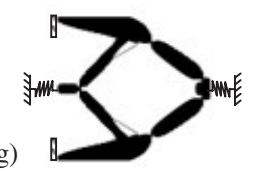

$k_{\text {in }}=k_{b} \times 10^{-4} \quad k_{\text {out }}=k_{b} \times 10^{-2}$

$k_{\text {in }}=k_{b} \times 10^{-2} \quad k_{\text {out }}=k_{b}$

(i)

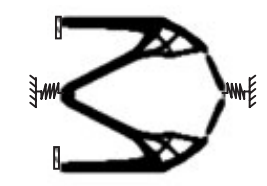

$k_{\text {in }}=k_{b} \quad k_{\text {out }}=k_{b} \times 10^{-2}$

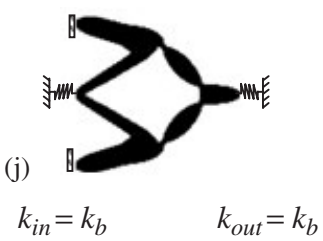

Figure 7. (a) Schematic of the force-inverter mechanism design problem; and (b)-(j) various design solutions of P1 obtained by maximizing mutual potential energy (MPE) with aluminium, and with different artificial spring stiffnesses on the I/P and $\mathrm{O} / \mathrm{P}$ of the mechanism.

imposed on all of the designs. Figure 7(b) shows the resulting material layout of a design obtained by solving the optimization problem of Equation (26) with artificial spring stiffnesses $k_{\text {in }}=k_{\text {out }}=10^{-4} \cdot k_{b}$. Clearly, the material layout throughout the model of the device is not continuous and contains numerous de facto hinge regions. Although the computed performance characteristics of devices such as that shown in Figure 7(b) (Table I) can sometimes appear to be remarkably good with large GA and complimentary compliance values, it must be borne in mind, that the design itself is so fragile that it would fail almost immediately upon being loaded. Thus the computed performance characteristics of compliant mechanisms that rely on de facto hinge regions are not necessarily achievable. The remaining material layout designs in Figure 7(c)-(j) are obtained either by using stiffer artificial springs on the input port, stiffer springs on the output port, or both. It bears mentioning that all of the intermediate designs shown in Figure 7(b)-(i) feature de facto hinge regions, and that only the design shown in 
Table I. Computed performance characteristics of selected continuous inverter designs and a hinged design at finite deformation.

\begin{tabular}{|c|c|c|c|c|c|c|}
\hline $\begin{array}{l}\text { Mechanism } \\
\text { description }\end{array}$ & $\begin{array}{l}\text { Workpiece } \\
\text { spring } \\
\text { stiffness }\end{array}$ & $\begin{array}{c}\text { Mean } \\
\text { compliance } \\
\Pi(\mathrm{Nm})\end{array}$ & $\begin{array}{l}\text { Complementary } \\
\text { compliance } E_{C} \\
\qquad(\mathrm{Nm})\end{array}$ & GA & MA & ME \\
\hline Polysilicon & $K=10^{5} \mathrm{~N} / \mathrm{m}$ & $7.73 \times 10^{-6}$ & $7.43 \times 10^{-6}$ & 0.962 & $7.44 \times 10^{-5}$ & $7.15 \times 10^{-5}$ \\
\hline Figure $8(a),(b)$ & $K=10^{6} \mathrm{~N} / \mathrm{m}$ & $7.73 \times 10^{-6}$ & $7.43 \times 10^{-6}$ & 0.962 & $7.44 \times 10^{-4}$ & $7.15 \times 10^{-4}$ \\
\hline$C=0.30$ & $K=10^{7} \mathrm{~N} / \mathrm{m}$ & $7.73 \times 10^{-6}$ & $7.43 \times 10^{-6}$ & 0.962 & $7.44 \times 10^{-3}$ & $7.15 \times 10^{-3}$ \\
\hline Aluminium & $K=10^{5} \mathrm{~N} / \mathrm{m}$ & $8.85 \times 10^{-6}$ & $1.74 \times 10^{-5}$ & 0.988 & $1.74 \times 10^{-4}$ & $1.72 \times 10^{-4}$ \\
\hline Figure $8(\mathrm{c}),(\mathrm{d})$ & $K=10^{6} \mathrm{~N} / \mathrm{m}$ & $8.84 \times 10^{-6}$ & $1.74 \times 10^{-5}$ & 0.988 & $1.74 \times 10^{-3}$ & $1.72 \times 10^{-3}$ \\
\hline$C=0.30$ & $K=10^{7} \mathrm{~N} / \mathrm{m}$ & $8.58 \times 10^{-6}$ & $1.70 \times 10^{-5}$ & 0.977 & $1.70 \times 10^{-2}$ & $1.67 \times 10^{-2}$ \\
\hline Nylon & $K=10^{5} \mathrm{~N} / \mathrm{m}$ & $4.84 \times 10^{-4}$ & & 1.011 & $4.90 \times 10^{-3}$ & $4.95 \times 10^{-3}$ \\
\hline Figure $8(e),(f)$ & $K=10^{6} \mathrm{~N} / \mathrm{m}$ & $4.62 \times 10^{-4}$ & $62 \times 10^{-4}$ & 1.001 & $4.63 \times 10^{-2}$ & $4.63 \times 10^{-2}$ \\
\hline$C=0.30$ & $K=10^{7} \mathrm{~N} / \mathrm{m}$ & $3.34 \times 10^{-4}$ & $3.08 \times 10^{-4}$ & 0.923 & $3.08 \times 10^{-1}$ & $2.84 \times 10^{-1}$ \\
\hline Aluminium & $K=10^{5} \mathrm{~N} / \mathrm{m}$ & $1.42 \times 10^{-4}$ & $2.69 \times 10^{-4}$ & 0.947 & $2.69 \times 10^{-3}$ & $2.54 \times 10^{-3}$ \\
\hline Figure $8(\mathrm{~g}),(\mathrm{h})$ & $K=10^{6} \mathrm{~N} / \mathrm{m}$ & & -4 & 0.945 & $2.63 \times 1$ & $2.47 \times 10^{-2}$ \\
\hline$C=0.10$ & $K=10^{7} \mathrm{~N} / \mathrm{m}$ & $9.11 \times 10^{-5}$ & $2.13 \times 10^{-4}$ & 0.935 & $2.30 \times 10^{-1}$ & $1.98 \times 10^{-1}$ \\
\hline Aluminium & $K=10^{5} \mathrm{~N} / \mathrm{m}$ & $3.57 \times 10^{-3}$ & $7.88 \times 10^{-3}$ & 1.0 & $7.81 \times 10^{-2}$ & $7.83 \times 10^{-2}$ \\
\hline Figure $8(\mathrm{i}),(\mathrm{j})$ & $K=10^{6} \mathrm{~N} / \mathrm{m}$ & & & 1.00 & -1 & $4.54 \times 10^{-1}$ \\
\hline$C=0.03$ & $K=10^{7} \mathrm{~N} / \mathrm{m}$ & $8.13 \times 10^{-5}$ & $8.83 \times 10^{-4}$ & 0.94 & $8.85 \times 10^{-1}$ & $8.27 \times 10^{-1}$ \\
\hline Aluminium & $K=10^{5} \mathrm{~N} / \mathrm{m}$ & $3.48 \times 10^{-3}$ & $1.22 \times 10^{-2}$ & 1.43 & $1.22 \times 10^{-1}$ & $1.74 \times 10^{-1}$ \\
\hline Figure $8(\mathrm{k}),(\mathrm{l})$ & $K=10^{6} \mathrm{~N} / \mathrm{m}$ & $5.60 \times 10^{-4}$ & $4.73 \times 10^{-3}$ & 1.40 & $4.73 \times 10^{-1}$ & $6.63 \times 10^{-1}$ \\
\hline$C=0.30$ & $K=10^{7} \mathrm{~N} / \mathrm{m}$ & $4.82 \times 10^{-5}$ & $6.64 \times 10^{-4}$ & 1.23 & $6.65 \times 10^{-1}$ & $8.20 \times 10^{-1}$ \\
\hline
\end{tabular}

Note: No springs were attached to the input port in the computations reported, and three different workpiece spring stiffnesses were used on the output port. The characteristics of each mechanism are reported for each of the workpiece stiffnesses considered under an actuation force of $100 \mathrm{~N}$.

Figure 7(j), which was obtained by using artificial spring stiffnesses $k_{\text {in }}=k_{\text {out }}=k_{b}$, is a continuous, monolithic design free of de facto hinge regions. It should be clear from the examples presented here that the stiffnesses of the artificial springs attached to the input and output ports of the devices have a very significant effect on the material continuity and preclusion of de facto hinges.

For a given structural material and a given material usage constraint, continuous, monolithic designs such as that shown in Figure 7(j) generally feature much smaller complimentary compliances than the family of designs that contain de facto hinges (Table I). In particular, the complimentary compliances of the hinge-free device shown in Figure 7(j) with workpiece springs attached to the $\mathrm{O} / \mathrm{P}$ are orders of magnitude smaller than those of devices that feature de facto hinges. In order to obtain continuous, hinge-free compliant mechanism designs that have higher compliance characteristics, the designer can resort to using more compliant structural materials, or to imposing much more restrictive material usage constraint values, or both. These issues are examined below. 
5.2.2. Effects of material properties and usage constraints. To explore the effects of material stiffness and material usage constraints on compliant mechanism characteristics, the inverter mechanism design problem P1 is solved once again with different structural materials and varied material usage constraints. When the inverter design problem is solved with a material usage constraint value $C=0.3$ and with structural materials of polysilicon, aluminium, and then nylon, the designs shown in Figures 8(a), (c), and (e), respectively, are obtained, and all exhibit material continuity. The corresponding deformed configurations of these three mechanisms, shown in Figures 8(b), (d), and (f) indicate that each functions without any de facto hinges. The computed performance characteristics of these mechanism designs when working against workpiece springs (Table I) indicate that while all have GA values in the neighbourhood of unity, the mechanisms with more compliant material have higher complimentary compliances and mechanical advantages.

Sparse designs were also obtained by solving the optimization problem P1 of Equation (26) with aluminium structural material, bounding values for the artificial springs on the input and output ports, and more restrictive material usage constraints. With a material usage constraint $C=0.10$, the design shown in Figures $8(\mathrm{~g})$, (h) was obtained, and with $C=0.03$, the design of Figures 8(i), (j) was obtained. The respective deformed configurations of these designs show the mechanisms functioning with well-distributed elastic deformation and no de facto hinges. Due to the slenderness of the individual members comprising the designs of Figures $8(\mathrm{~h})$ and (j), it is tempting to interpret them as pin-jointed truss structures similar to those obtained in the work of Reference [4]. The obvious curvature in the structural members of the device designs under loading (Figures 8(h) and (j)) clearly indicates, however, that the joint regions are continuous and transmit bending moments. The performance characteristics of the sparse, hinge-free, aluminium designs, reported in Table I are further noteworthy in that with increasing sparsity, the complimentary compliance and mechanical efficiency characteristics of the designs increase dramatically.

The computed performance characteristics of the hinged aluminium design of Figure 7(b) and Figure 8(k), (l) (Table I) indicate that its performance metrics (GA, MA, $E_{C}$ ) are typically greater than those of continuous hinge-free mechanisms. It is re-emphasized, however, that the hinged mechanism of Figures 7(b) and 8(k), (l), being discontinuous and using hinges, is not physically realizeable as a compliant mechanism. The most sparse $(C=0.03)$ hingefree monolithic aluminium compliant mechanism considered (Figure 8(i), (j)), has performance characteristics most comparable to those of the hinged aluminium device.

Since a threshold complimentary compliance $E_{C}^{*}$ of the mechanism is generally desired when working against an anticipated level of workpiece resistance, the extended design problem P2 must typically be solved to obtain the infimum (greatest lower bound) of the material usage constraint $C$. To illustrate, problem $\mathrm{P} 1$ for the inverter mechanism was solved a number of times with stiff artificial springs utilizing different material usage constraints $(C=0.03,0.07$, $0.1,0.15,0.2,0.25$, and 0.3 ). For each design solution of P1, finite deformation hyperelastic analysis was performed to compute the complimentary compliance of the mechanism design under a $100 \mathrm{~N}$ actuation force applied to its input port. For three different workpiece resistances $k_{\text {workpiece }}\left(10^{5} \mathrm{~N} / \mathrm{m} ; 10^{6} \mathrm{~N} / \mathrm{m} ; 10^{7} \mathrm{~N} / \mathrm{m}\right.$; ) the complimentary compliances vs material usage constraint $C$ were computed (Figure 9). From graphs of this type it is thus possible to select material usage constraints for given workpiece resistance that will yield the desired complimentary compliance. To illustrate, for $k_{\text {workpiece }}=10^{5} \mathrm{~N} / \mathrm{m}$ and $f_{\text {in }}=100 \mathrm{~N}$, if a desired complimentary compliance of the force-inverter mechanism of $2 \times 10^{-4} \mathrm{~J}$ were required, the 
(a)

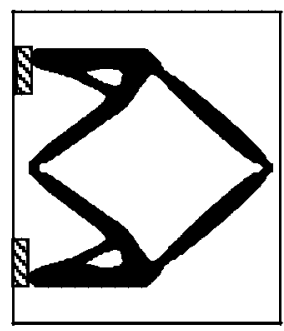

(b)

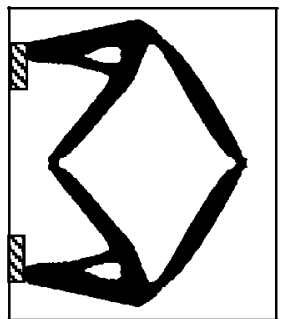

(g)

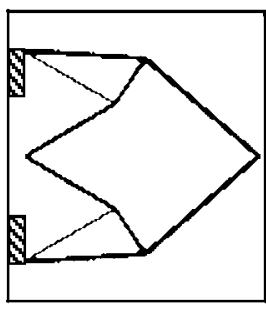

(h)

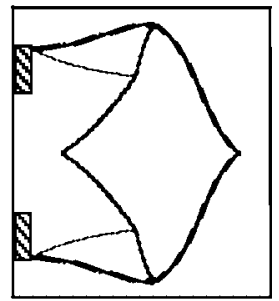

(c)

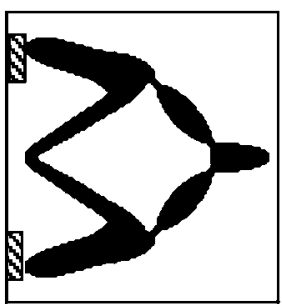

(d)

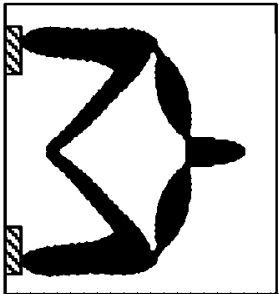

(i)

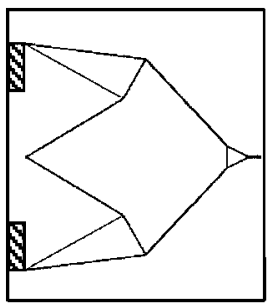

(j)

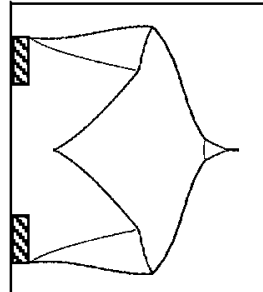

(e)

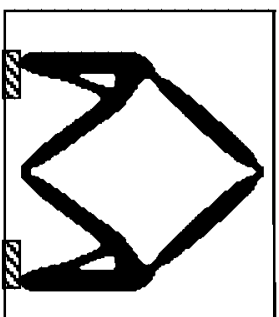

(f)

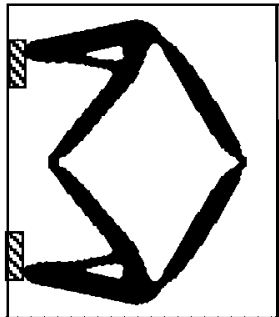

(k)

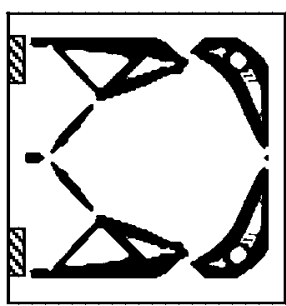

(1)

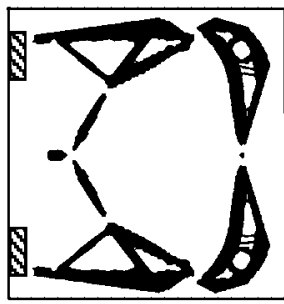

Figure 8. Inverter mechanism design solutions of $\mathrm{P} 1$ achieved with different materials and material usage constraints. For the designs shown in (a)-(j) with each structural material used, the corresponding baseline spring stiffnesses were used for the input and output port springs: (a) polysilicon design $[C=0.30]$; (b) deformed configuration; (c) aluminium design $[C=0.30]$; (d) deformed configuration; (e) nylon design $[C=0.30]$; (f) deformed configuration; (g) aluminium design $[C=0.10]$; (h) deformed configuration; (i) aluminium design $[C=0.03]$; (j) deformed configuration; (k) hinged aluminium design $[C=0.30]$ achieved with $k_{\text {in }}=k_{\text {out }}=10^{-4} k_{b}$; and (l) deformed configuration.

greatest lower bound on $C$ (infimum) for which this could be obtained is a value of 0.11 . To further illustrate, for $k_{\text {workpiece }}=10^{6} \mathrm{~N} / \mathrm{m}$ and $f_{\text {in }}=100 \mathrm{~N}$, if $E_{C}^{*}=2 \times 10^{-3} \mathrm{~J}$ then inf $C=0.05$. 


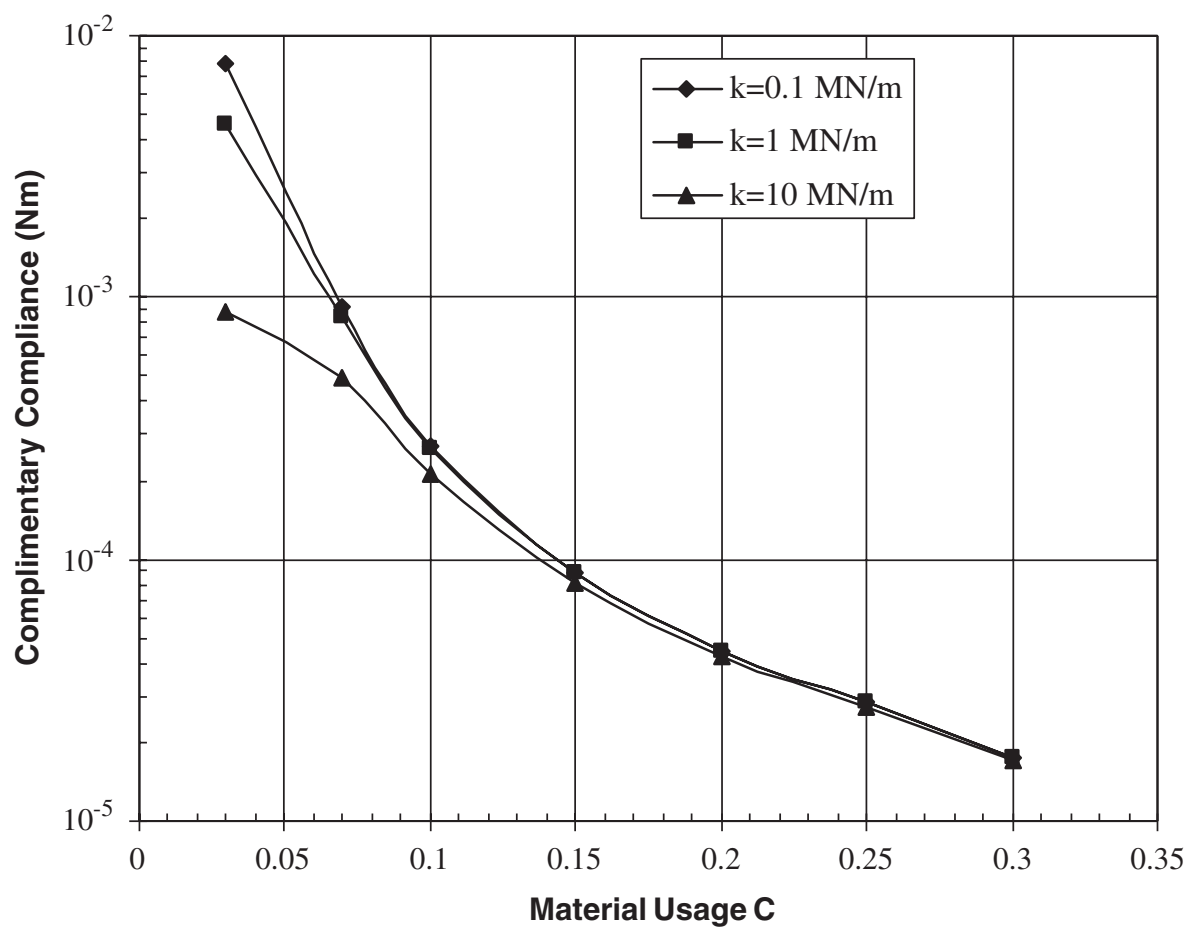

Figure 9. Inverter mechanism's computed complimentary compliance at finite deformation vs material usage factor $C$ for different workpiece spring stiffnesses. The mechanism material is aluminium.

5.2.3. Production model investigation of sparse inverter designs. To further investigate the sparse aluminium mechanism designs of Figures $8(\mathrm{~g})$, (i) the material layouts were finely remeshed from grid-type meshes used in continuum topology optimization to more refined conforming meshes of six-noded biquadratic triangular elements (Figure 10) using the techniques described in Reference [30]. The mechanism models that utilize the more refined conforming meshes are here termed 'production models.' Due to the sparsity of the model designs with $C=0.03$ and 0.10 and the associated slenderness of structural members, the possibilities of buckling and material failure within the mechanism cannot be neglected. The output ports of these production models were restrained (Figures 10(a), (d)) and the buckling loads and modes were computed (Figures 10(b), (e)) using linearized buckling eigenmode analysis [28,31]. Hyperelastic geometrically non-linear analysis was also performed on the production models, loading them at the mechanism input ports until the output ports went through displacements of $3 \mathrm{~mm}$ at the output ports. The input forces required to generate the $3 \mathrm{~mm}$ output port displacements were significantly smaller than the respective computed buckling loads for these mechanisms. The peak computed von Mises stresses $\left(\sqrt{J_{2}}\right)$ in the mechanism production models at loads that generate $3 \mathrm{~mm}$ output port displacements in Figures $10(\mathrm{c})$ and (f) are $31 \mathrm{MPa}$ in the more sparse model $(C=0.03)$ and $71 \mathrm{MPa}$ in the less sparse design $(C=0.10)$. These peak computed generalized shear stresses are below the yield stresses of many aluminium alloys. 


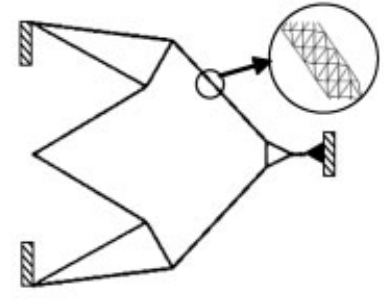

(a)

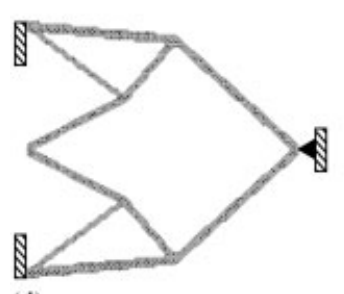

(d)

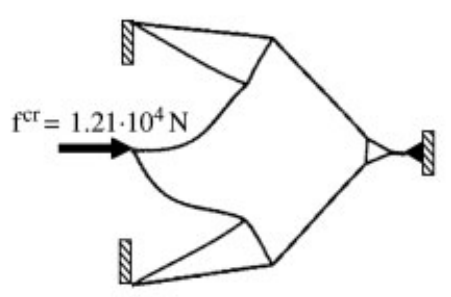

(b)

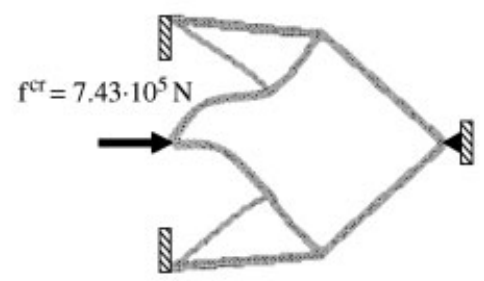

(e)
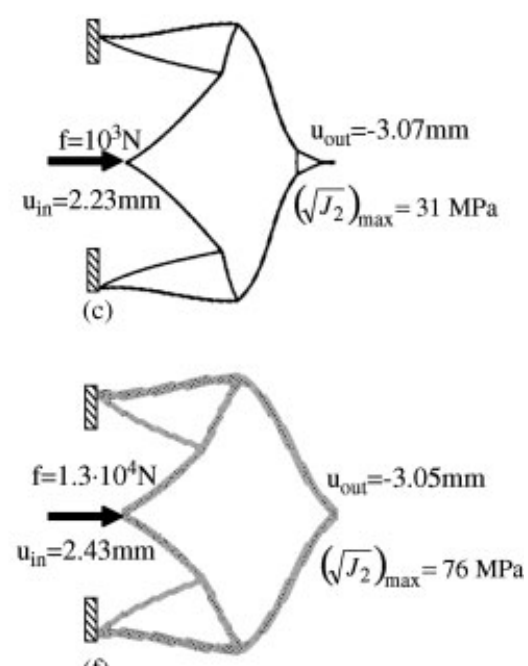

(f)

Figure 10. Production model analyses of sparse, aluminium inverter designs: (a) mesh of the design with $C=0.03$; (b) computed buckling mode and force with the O/P restrained; (c) geometrically non-linear response to input force that generates $3 \mathrm{~mm}$ output displacement with no workpiece resistance; (d) mesh of the design with $C=0.10$; (e) buckling mode and force with $\mathrm{O} / \mathrm{P}$ restrained; and

(f) response to input force that generates $3 \mathrm{~mm}$ output displacement with no workpiece resistance.

Production model analysis of the compliant mechanism designs is also noteworthy for the fact that a straight algorithmic translation of the topology design models into production models was performed without any intelligent decisions required. The production force inverter model based on $C=0.03$ (Figure 10) has a computed finite deformation GA $=1.38$, whereas the production model based on $C=0.10$ has $\mathrm{GA}=1.26$ at the level of deformation shown. That the computed performance characteristics of the mechanism production models equal or exceed those of the topology design models confirms the absence of any de facto hinges in the topology design models which would artificially boost their computed performance characteristics.

\subsection{Gripper mechanism designs}

It is fair and reasonable to ask whether or not the trends observed in design of the inverter mechanism hold when designing other mechanisms having different objectives, loading conditions, and boundary restraints. Here this issue is partially explored by considering the gripper mechanism design problem shown in Figure 11(a). The general design objective is to achieve a mechanism such that when a horizontal force is applied at its input port, the opposing output ports move vertically to pinch and thus grip a workpiece. The mechanism is designed with aluminium, and the bounding spring stiffness for the size and support conditions shown is $k_{b}=1.6 \times 10^{10} \mathrm{~N} / \mathrm{m}$.

The design problem P1 was solved with nine combinations of artificial springs $k_{\text {in }}$ and $k_{\text {out }}$ attached to the input and output ports. For each combination of $k_{\text {in }}$ and $k_{\text {out }}$ the design problem was solved to maximize the MPE subject to a material usage constraint $(C=0.30)$. Many of the design solutions shown in Figures 11(b)-(j) feature numerous de facto hinges and 


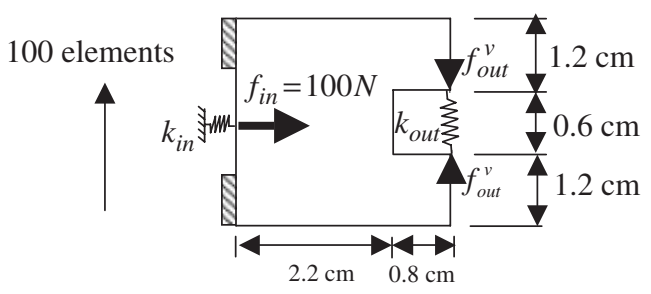

(a)
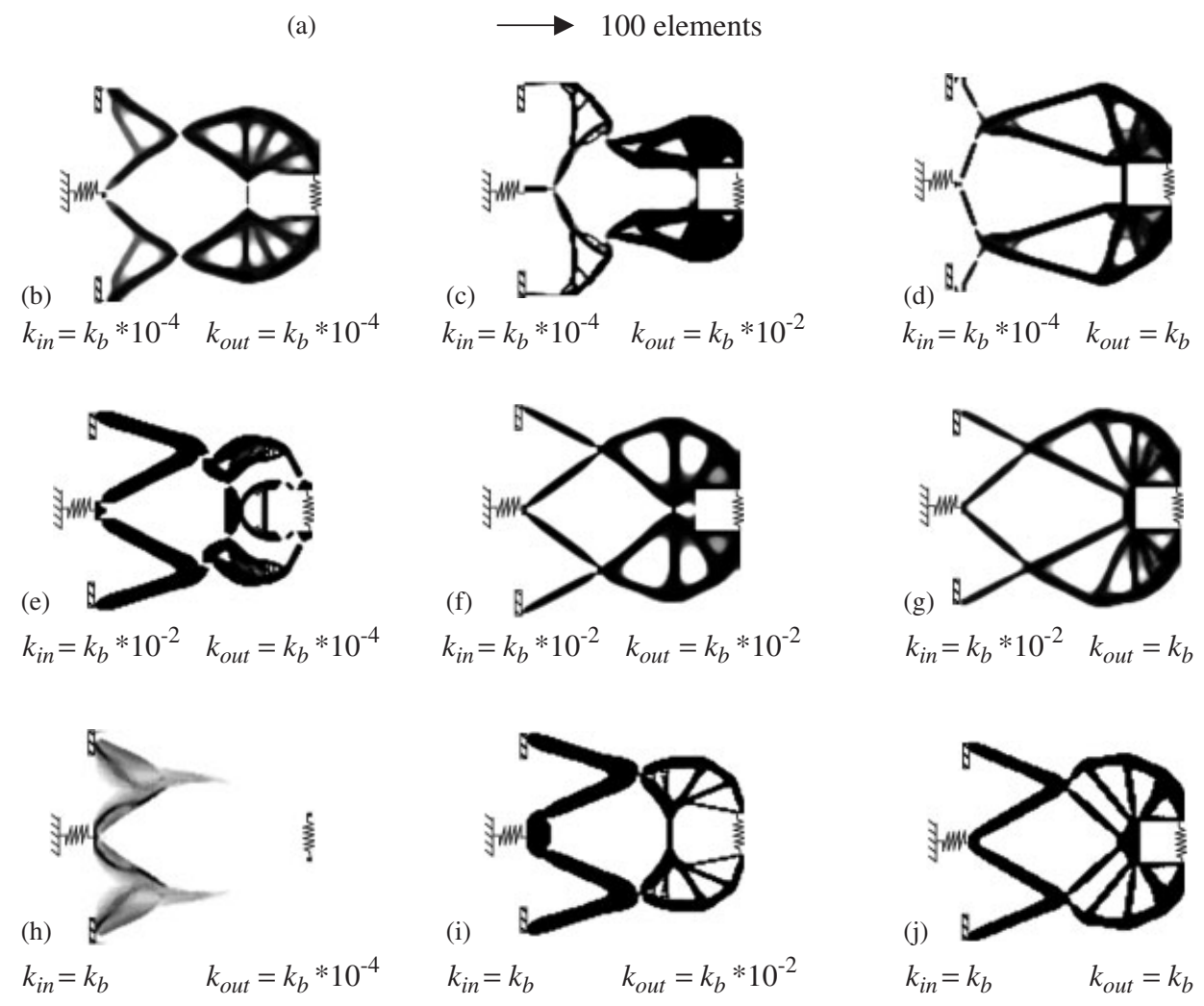

Figure 11. (a) Schematic of the gripper mechanism design problem; and (b)-(j) various design solutions of problem P1 obtained by maximizing mutual potential energy (MPE) with aluminium, $C=0.30$, and with different artificial spring stiffnesses on the I/P and $\mathrm{O} / \mathrm{P}$ of the mechanism.

lack material continuity when the artificial springs are much smaller than the bounding spring value. As the artificial spring values on both ports approach the bounding values, however, the discontinuities and de facto hinges eventually vanish. The design shown in Figures 11(j) was achieved with the artificial springs having upper bound values attached to both the input and output ports and is noteworthy for having no de facto hinges.

Since the hinge-free monolithic design of Figure 11(j) is quite stiff, the achievement of more compliant (but still hinge-free) designs can be achieved by solving P1 with more restrictive material usage constraints or by solving the problem with different structural materials. The gripper mechanism design problem was thus solved with different structural materials 
(a)

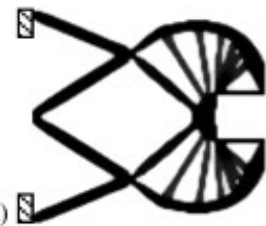

(b)

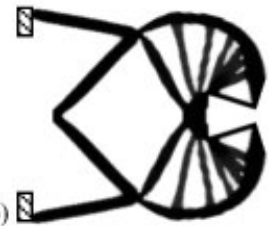

(g)

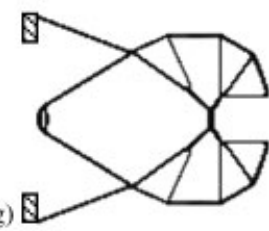

(h)

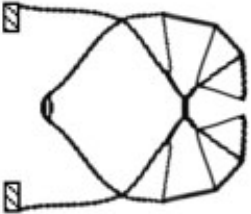

(c)

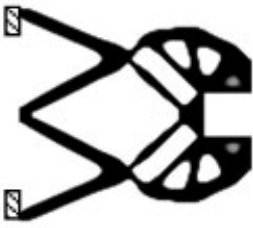

(d)

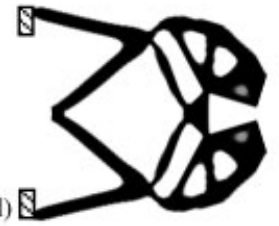

(i)

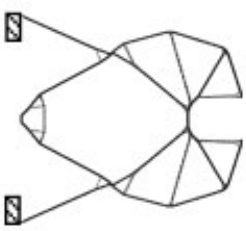

(i)

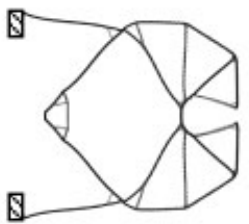

(e)

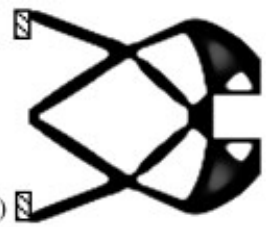

(f)

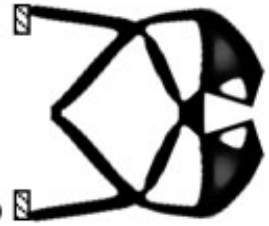

(k)

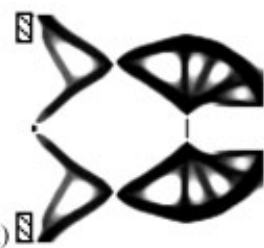

(1)

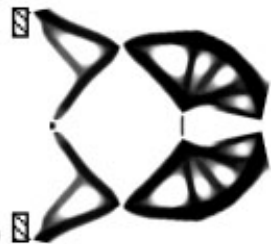

Figure 12. Various gripper mechanism solutions of P1 achieved with different materials and material usage constraints. For the designs shown in (a)-(j) with each structural material used, the corresponding baseline spring stiffnesses were used for the input and output port springs: (a) polysilicon design with $C=0.30$; (b) deformed configuration; (c) aluminium design with $C=0.30$; (d) deformed configuration; (e) nylon design with $C=0.30$; (f) deformed configuration; (g) aluminium design with $C=0.10$; (h) deformed configuration; (i) aluminium design with $C=0.03$; (j) deformed configuration; (k) hinged aluminium design achieved with $C=0.30$ and $k_{\text {in }}=k_{\text {out }}=10^{-4} k_{b}$; and (l) deformed configuration.

(polysilicon and nylon) and different material usage constraints, with the designs shown in Figure 12 and the tabulated performance characteristics in Table II. With the exception of those shown in Figure 12(k) and (l) all of the designs in Figure 12 were obtained by solving P1 with upper bound artificial spring stiffnesses. It is noteworthy that all of the P1 design solutions obtained with stiff artificial springs are continuous and free of de facto hinges.

The complimentary compliances of the aluminium gripper mechanisms at finite deformation with only workpiece springs on the output ports and no springs on the input ports are shown in Figure 13. As is expected, the complimentary compliances of the mechanisms increase quite dramatically as the material usage constraints values $(C)$ are reduced, or as the sparsity of the mechanisms is increased. Accordingly, for target values $E_{C}^{*}$ of the gripper mechanism's 
Table II. Computed performance characteristics at finite deformation of selected continuous gripper designs and a hinged design.

\begin{tabular}{|c|c|c|c|c|c|c|}
\hline $\begin{array}{l}\text { Mechanism } \\
\text { description }\end{array}$ & $\begin{array}{l}\text { Workpiece } \\
\text { spring } \\
\text { stiffness }\end{array}$ & $\begin{array}{c}\text { Mean } \\
\text { compliance } \\
\Pi(\mathrm{N} \mathrm{m})\end{array}$ & $\begin{array}{c}\text { Complementary } \\
\text { compliance } E_{C} \\
(\mathrm{~N} \mathrm{~m})\end{array}$ & GA & MA & ME \\
\hline Polysilicon & $K=10^{5} \mathrm{~N} / \mathrm{m}$ & $4.58 \times 10^{-6}$ & $1.29 \times 10^{-6}$ & 1.408 & $6.45 \times 10^{-5}$ & $9.08 \times 10^{-5}$ \\
\hline Figure $12(a),(b)$ & $K=10^{6} \mathrm{~N} / \mathrm{m}$ & $4.58 \times 10^{-6}$ & $1.29 \times 10^{-6}$ & 1.408 & $6.45 \times 10^{-4}$ & $9.08 \times 10^{-4}$ \\
\hline$C=0.30$ & $K=10^{7} \mathrm{~N} / \mathrm{m}$ & $4.58 \times 10^{-6}$ & $1.29 \times 10^{-6}$ & 1.408 & $6.45 \times 10^{-3}$ & $9.08 \times 10^{-3}$ \\
\hline Aluminium & $K=10^{5} \mathrm{~N} / \mathrm{m}$ & $1.26 \times 10^{-5}$ & $3.58 \times 10^{-5}$ & 1.417 & $1.79 \times 10^{-4}$ & $2.54 \times 10^{-4}$ \\
\hline Figure $12(\mathrm{c}),(\mathrm{d})$ & $K=10^{6} \mathrm{~N} / \mathrm{m}$ & $1.26 \times 10^{-5}$ & $3.58 \times 10^{-5}$ & 1.417 & $1.79 \times 10^{-3}$ & $2.54 \times 10^{-3}$ \\
\hline$C=0.30$ & $K=10^{7} \mathrm{~N} / \mathrm{m}$ & $1.20 \times 10^{-5}$ & $3.46 \times 10^{-5}$ & 1.404 & $1.74 \times 10^{-2}$ & $2.44 \times 10^{-2}$ \\
\hline Nylon & $K=10^{5} \mathrm{~N} / \mathrm{m}$ & $3.39 \times 10^{-4}$ & $9.24 \times 10^{-4}$ & 1.354 & $4.62 \times 10^{-3}$ & $6.25 \times 10^{-3}$ \\
\hline Figure 12(e), (f) & $K=10^{6} \mathrm{~N} / \mathrm{m}$ & $3.04 \times 10^{-4}$ & $8.64 \times 10^{-4}$ & 1.329 & $4.33 \times 10^{-2}$ & $5.75 \times 10^{-2}$ \\
\hline$C=0.30$ & $K=10^{7} \mathrm{~N} / \mathrm{m}$ & $1.50 \times 10^{-4}$ & $5.28 \times 10^{-4}$ & 1.197 & $2.64 \times 10^{-1}$ & $3.16 \times 10^{-1}$ \\
\hline Aluminium & $K=10^{5} \mathrm{~N} / \mathrm{m}$ & $1.79 \times 10^{-4}$ & $6.04 \times 10^{-4}$ & 1.668 & $3.00 \times 10^{-3}$ & $5.00 \times 10^{-3}$ \\
\hline Figure $12(\mathrm{~g}),(\mathrm{h})$ & $K=10^{6} \mathrm{~N} / \mathrm{m}$ & $1.64 \times 10^{-4}$ & $5.76 \times 10^{-4}$ & 1.664 & $2.90 \times 10^{-2}$ & $4.83 \times 10^{-2}$ \\
\hline$C=0.10$ & $K=10^{7} \mathrm{~N} / \mathrm{m}$ & $2.26 \times 10^{-4}$ & $3.97 \times 10^{-4}$ & 1.633 & $1.90 \times 10^{-1}$ & $3.10 \times 10^{-1}$ \\
\hline Aluminium & $K=10^{5} \mathrm{~N} / \mathrm{m}$ & $5.60 \times 10$ & $1.85 \times 10$ & 1.443 & $9.24 \times 1$ & $1.33 \times 10^{-1}$ \\
\hline Figure 12(i), (j) & $K=10^{6} \mathrm{~N} / \mathrm{m}$ & $1.37 \times 10^{-3}$ & $8.34 \times 10^{-3}$ & 1.354 & $4.18 \times 10^{-1}$ & $5.66 \times 10^{-1}$ \\
\hline$C=0.03$ & $K=10^{7} \mathrm{~N} / \mathrm{m}$ & $2.55 \times 10^{-4}$ & $1.29 \times 10^{-3}$ & 0.960 & $6.48 \times 10^{-1}$ & $6.22 \times 10^{-1}$ \\
\hline Alumi & $K=10^{5} \mathrm{~N} / \mathrm{m}$ & $5.07 \times 10^{-3}$ & $1.76 \times 10^{-2}$ & 1.517 & $8.80 \times 10^{-2}$ & $1.33 \times 10^{-1}$ \\
\hline Figure $12(\mathrm{k}),(\mathrm{l})$ & $K=10^{6} \mathrm{~N} / \mathrm{m}$ & $1.07 \times 10^{-3}$ & $8.04 \times 10^{-3}$ & 1.497 & $4.02 \times 10^{-1}$ & $6.00 \times 10^{-1}$ \\
\hline$C=0.30$ & $K=10^{7} \mathrm{~N} / \mathrm{m}$ & $5.82 \times 10^{-5}$ & $1.24 \times 10^{-3}$ & 1.398 & $6.21 \times 10^{-1}$ & $8.66 \times 10^{-1}$ \\
\hline
\end{tabular}

Note: No springs were attached to the input port in the computations reported, and three different workpiece spring stiffnesses were used on the output port. The characteristics of each mechanism are reported for each of the three workpiece stiffnesses considered under an actuation force of $100 \mathrm{~N}$.

complimentary compliance under specified input forces and output port resistances, the appropriate material usage factors can be determined.

\section{DISCUSSION AND CONCLUSIONS}

In the examples of the preceding section design problem P1 was solved a number of times for both a force-inverter mechanism and a gripper mechanism. For demonstrative purposes, each of the P1 design problems was solved for a fixed material usage constraint value $(C=0.3)$ with nine different combinations of artificial spring stiffnesses on the input and output ports (Figures 7 and 11). The results demonstrate that when problem P1 is solved with artificial springs of relatively low stiffness $\left(k \ll k_{b}\right)$, compliant mechanism designs featuring varying amounts of $d e$ facto hinges are obtained. The results further indicate that when problem P1 is solved with artificial springs of appropriate stiffness $\left(k=k_{b}\right)$ compliant mechanism designs free of de facto hinges are obtained. Differences in the performance characteristics of mechanisms containing 


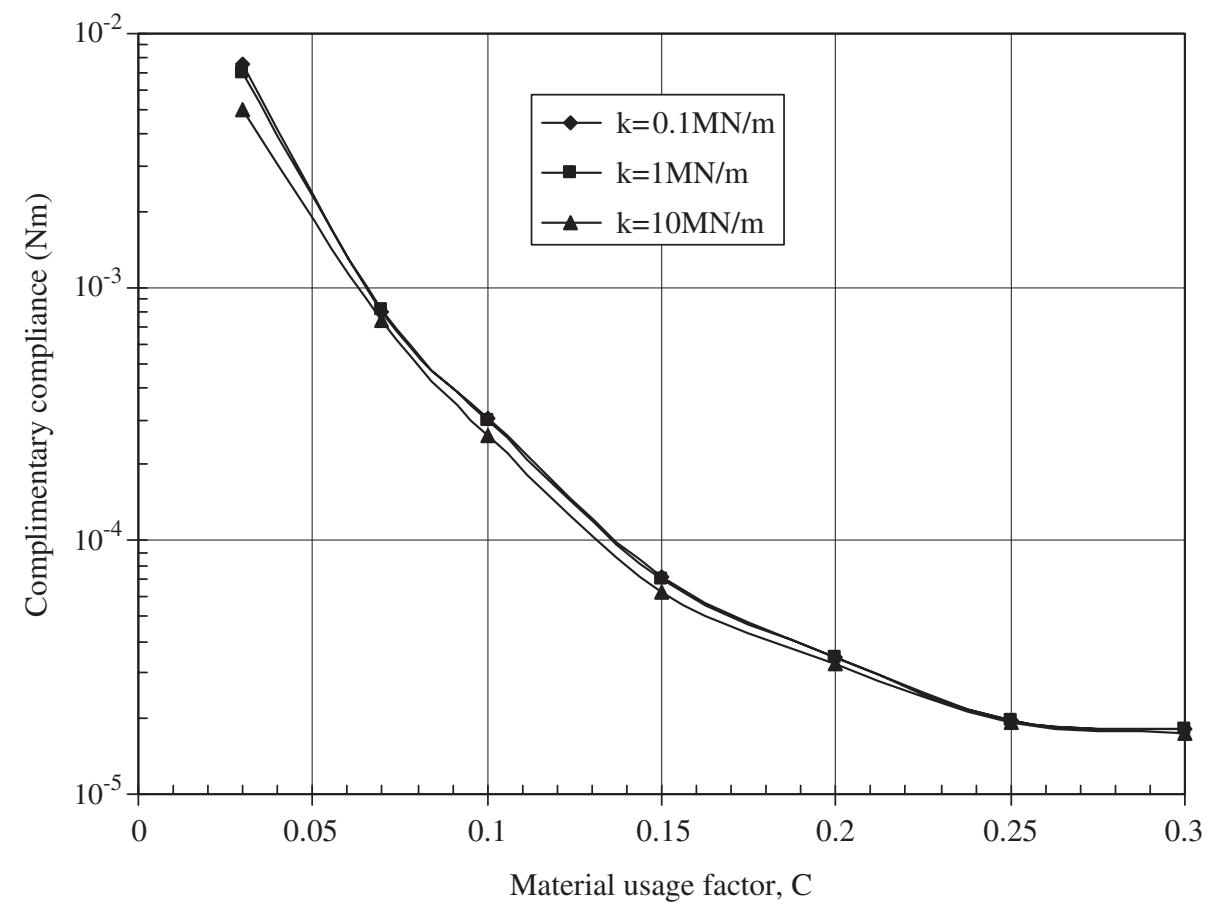

Figure 13. Computed complimentary compliance at finite deformation vs material usage factor $C$ for the gripper mechanism when made of aluminium. Three curves are shown for different workpiece spring stiffnesses.

de facto hinges and those obtained herein that are free of such hinges can be seen in related videos showing the mechanisms undergo cyclic loadings [32].

For a given amount of structural material, the hinge-free design solutions emerging from P1 tend to be much stiffer (e.g. having much smaller complimentary compliances when working against workpiece resistances) than those that feature de facto hinges. Nevertheless, hinge-free compliant mechanism designs having large finite deformation complimentary compliances were shown to be achievable herein by solving an extended problem P2 that finds the infimum of material usage constraint values on the interval $(0,1)$. The resulting compliant mechanism designs emerging from P2 were found to be sparse but completely free of de facto hinges. Such compliant mechanism designs feature elastic deformation that is well-distributed throughout the mechanism rather than concentrated in a few specific hinge regions [32]. Since the extended problem P2 involves a search in $\mathfrak{R}^{1}$, the computational effort required to solve P2 is typically only five to ten times that required to solve P1 just once.

The sparse, hinge-free compliant mechanism designs obtained in this work are believed, in a practical sense that considers both manufacturability and durability, to be superior to the mechanism designs that contain de facto hinges. Furthermore, the computed kinematic performance characteristics of the hinge-free compliant mechanism designs have been found in the cases considered herein to be comparable to those of the hinged mechanisms as was shown in Tables I and II. 
For sparse designs, that is those with $C \ll 1$, very fine meshes are needed both to resolve the limited material layout distribution and to provide accurate analysis results. In the example problems presented above, the starting resolution for both the force-inverter and gripper problems was a uniform mesh of $100 \times 100$ bilinear continuum elements. For very sparse material usage, however, this mesh resolution was insufficient and so layout designs were mapped onto refined meshes of $200 \times 200$ and even $400 \times 400$ elements and the design process was continued. For highly refined meshes together with sparse material usage, great economy and computational efficiency are gained using analysis problem size reduction algorithms [28] in which empty elements are neglected and nodes in the void region of the structure are restrained.

The proposed design process has been demonstrated herein on the realization of an elastic force-inverter compliant mechanism and an elastic gripping compliant mechanism where the output port was designed to go through uni-directional motion either parallel to or perpendicular to the mechanism's axis of symmetry. There are many current applications, however, where the designer might seek to have the output port of the mechanism follow a certain curvilinear trajectory in response to a sequence of actuation forces while working against specified workpiece resistances at the output port. While such 'path-following' mechanisms can in principle be designed, they may not be very robust because, for even modest variations in the workpiece resistance, the mechanism $\mathrm{O} / \mathrm{P}$ may deviate quite significantly from the desired trajectory. A potentially more robust approach to obtaining finite deformation path-following compliant mechanisms that accounts for reasonable variations in workpiece resistances is to employ control algorithms [29] that solve for the sequence of actuation forces needed to obtain the desired path-following $\mathrm{O} / \mathrm{P}$ response of the compliant mechanism under a variety of workpiece resistances. Sparse flexible designs obtained from the current framework have so-far appeared quite controllable and well-suited to usage in the proposed approach.

\section{REFERENCES}

1. Howell LL, Midha A. A loop-closer theory for the analysis and synthesis of compliant mechanisms. Journal of Mechanical Design 1996; 118:121-125.

2. Howell LL. Compliant Mechanisms. Wiley: New York, 2001.

3. Ananthasuresh GK, Kota S, Gianchandani Y. A methodical approach to the design of compliant micromechanisms. Solid-State Sensor, Actuator Workshop, South Carolina, 1994; 13-16.

4. Frecker MI, Ananthasuresh GK, Nishiwaki S, Kikuchi N, Kota S. Topological synthesis of compliant mechanisms using multi-criteria optimization. Journal of Mechanical Design 1997; 119:238-245.

5. Larsen U, Sigmund O, Bouwstra S. Design and fabrication of compliant micro-mechanisms and structures with negative Poisson's ratio. Journal of Microelectromechanical Systems 1997; 6:99-106.

6. Bruns TE, Tortorelli DA. Topology optimization of geometrically nonlinear structures and compliant mechanisms. Computer Methods in Applied Mechanics and Engineering 2001; 190:3443-3459.

7. Buhl T, Pedersen W, Sigmund O. Stiffness design of geometrically nonlinear structures using topology optimization. Structural and Multidisciplinary Optimization 2000; 19:93-104.

8. Pedersen CBW, Buhl T, Sigmund O. Topology synthesis of large-displacement compliant mechanisms. International Journal for Numerical Methods in Engineering 2001; 50:2683-2705.

9. Saxena A, Ananthasuresh GK. Topology synthesis of compliant mechanisms for nonlinear force-deflection and curved path specifications. Journal of Mechanical Design (ASME) 2001; 123:33-42.

10. Duysinx P, Bendsoe O. Topology optimization of continuum structures with local stress constraints. International Journal for Numerical Methods in Engineering 1998; 43(2):1453-1478.

11. Saxena A, Ananthasuresh GK. Topology design of compliant mechanisms with strength consideration. Mechanics of Structures and Mechanisms 2003; 29(2):199-221.

12. Yin L, Ananthasuresh GK. Topology optimization of compliant mechanisms with multiple material using peak function material interpolation scheme. Structural and Multidisciplinary Optimization 2001; 23:49-62. 
13. Sigmund O. Design of multiphysics actuators using topology optimization-Part II: Two-material structures. Computer Methods in Applied Mechanics and Engineering 2001; 190:6605-6627.

14. Sigmund O. Optimum design of microelectromechanical systems. In Mechanics for a New Millennium, Aref H, Philips JW (eds). Kluwer: Dordrecht, 2001; pp. 505-520.

15. Chen BC, Silva ECN, Kikuchi N. Advances in computational design and optimization with application to MEMS. International Journal for Numerical Methods in Engineering 2001; 52:23-62.

16. Poulsen TA. Topology optimization in wavelet space. International Journal for Numerical Methods in Engineering 2003; 57:741-760.

17. Poulsen TA. A new scheme for imposing a minimum length scale in topology optimization. International Journal for Numerical Methods in Engineering 2002; 53:567-582.

18. Yin L, Ananthasuresh GK. Design of distributed compliant mechanisms. Mechanics Based Design of Structures and Machines 2003; 31(2):151-179.

19. Canfield S, Frecker M. Topology optimization of compliant mechanical amplifiers for piezoelectric actuators. Structural and Multidisciplinary Optimization 2000; 20:269-279.

20. Hetrick JA, Kikuchi N, Kota S. Robustness of compliant mechanism topology optimization formulations. Proc. SPIE International Symposium on Smart Structures and Materials, Mathematics and Control in Smart Structures 1999; 3667:244-254.

21. Saxena A, Ananthasuresh GK. On an optimal property of compliant topologies. Structural and Multidisciplinary Optimization 2000; 19:36-49.

22. Shield RT, Prager W. Optimal structural design for given deflection. Journal of Applied Mathematics and Physics 1970; 21:513-523.

23. Sigmund O. On the design of compliant mechanisms using topology optimization. Mechanics of Structures and Machines 1997; 25(4):495-526.

24. Haber RB, Jog CS, Bendsoe MP. A new approach to variable-topology shape design using a constraint on perimeter. Structured Optimization 1996; 11:1-12.

25. Rahmatalla S, Swan CC. A Q4/Q4 continuum structural topology optimization implementation. Structural and Multidisciplinary Optimization 2004; 27:130-135.

26. Swan CC, Kosaka I. Voigt-Reuss topology optimization for structures with linear elastic material behaviours. International Journal for Numerical Methods in Engineering 1997; 40:3033-3057.

27. Ciarlet PG. Mathematical Elasticity: Three-Dimensional Elasticity. vol. I. Elsevier: Amsterdam, 1988.

28. Rahmatalla S, Swan CC. Continuum topology optimization of buckling-sensitive structures. AIAA Journal 2003; 41(6):1180-1189.

29. Swan CC, Rahmatalla S. Topology design and control of path-following compliant mechanisms, paper AIAA-2004-4351 presented at 10th AIAA/ISSMO MAO Conference, Albany, New York, 2004.

30. Kim HJ, Swan CC. Automated meshing and unit cell analysis of periodic composites with hierarchical quadratic tetrahedral elements. International Journal for Numerical Methods in Engineering 2003; 58(11):1683-1711.

31. Rahmatalla S, Swan CC. Form-finding sparse structures with continuum topology optimization. Journal of Structural Engineering 2003; 129(12):1707-1716.

32. http://www.engineering.uiowa.edu/ swan/struct_opt/sopt.html 